livraisons

d'Histoire

de l'Architecture

\section{Livraisons de l'histoire de l'architecture}

28 | 2014

Jean-Louis Pascal

\title{
Après la bibliothèque
}

After the Library

Nach der Bibliothek

\section{Anne Richard-Bazire}

\section{(2) OpenEdition}

\section{Journals}

Édition électronique

URL : http://journals.openedition.org/lha/397

DOI : 10.4000/lha.397

ISSN : 1960-5994

Éditeur

Association Livraisons d'histoire de l'architecture - LHA

Édition imprimée

Date de publication : 10 décembre 2014

Pagination : 185-208

ISSN : $1627-4970$

Référence électronique

Anne Richard-Bazire, «Après la bibliothèque », Livraisons de l'histoire de l'architecture [En ligne], 28 | 2014, mis en ligne le 10 décembre 2016, consulté le 02 mai 2019. URL : http://

journals.openedition.org/lha/397 ; DOI : 10.4000//ha.397

Ce document a été généré automatiquement le 2 mai 2019.

Tous droits réservés à l'Association LHA 


\title{
Après la bibliothèque
}

\author{
After the Library \\ Nach der Bibliothek
}

Anne Richard-Bazire

1 La fin de la vie de Pascal est marquée par la mélancolie ${ }^{1}$. Il avait quitté avec peine la Bibliothèque nationale où il avait œuvré pendant trente-sept ans. Ce départ douloureux se double de ce qu'il considère comme une trahison : après le vol de la Joconde au Louvre, le 21 août 1911, une commission est nommée pour s'assurer de la protection de la Bibliothèque contre le vol et l'incendie. On demande à Pascal de modifier son programme élaboré en 1891. Il s'en ouvre à Louise Garnier :

« Je sors d'une longue séance de deux heures et demi où j'ai défendu pied à pied mon œuvre de vingt ans contre le sourire ironique de ceux qu'étonnent ces ardeurs d'artiste chez un vieillard, des parlementaires prétextant plutôt niaisement des économies, contre la crainte du fonctionnaire ou contre la sournoiserie de ceux qui visent une place à prendre, etc. Cette bataille m'a fait connaître de vilains dessous et le plus singulier est qu'avant la nomination de [cette] commission [...], rien ne pouvait me faire soupçonner qu'on désirât modifier le programme que j'ai lu ce matin, signé de Deschanel et remontant à 1891. Vous voulez bien comparer ma peine à l'émotion de mon grand chef lorsqu'il s'est agi de saboter son Monte Carlo² : toutes proportions gardées vous pouvez juger en effet de ma déconvenue alors que j'espérais avoir fait une œuvre qui ne fût pas indigne des maîtres qui m'ont honoré de leur direction et de leur amitié3. »

Ce qui va aider Pascal dans cette fin de vie solitaire, c'est son rôle de chef d'atelier; les questions liées à l'éducation le passionnent toujours et il va participer à la création des écoles régionales d'architecture. Pascal s'oppose à l'uniformité des styles; il en avait fait son cheval de bataille alors que, membre temporaire du conseil des bâtiments civils en 1880-1881 puis inspecteur général à partir de 1889, il se battait pour que soit laissée aux architectes la possibilité de développer ce que Georges Seure appelle «la fantaisie raisonnée, mais créatrice $»^{4}$. L'internationalisation des styles due à la multiplication des revues et à la propagation des modèles grâce à la photographie, au développement des transports, à l'industrialisation des moyens de production des matériaux qui en abaisse le coût, aux règles de plus en plus restrictives de l'urbanisme, telles que Georges Haussmann 
en avait mis en œuvre à Paris, à la densification des villes et au développement de l'hygiénisme, Charles Garnier et Auguste Ammann (1844-1921) l'avaient théorisée dans L'Habitation humaine en 1892, affirmant que «le pittoresque et le caractère individuel n'ont plus de place dans les constructions d'aujourd'hui $»^{5}$. Contre ce phénomène, JeanLouis Pascal prônera l'usage des matériaux locaux et la mise en valeur des particularismes régionaux, s'affirmant comme un architecte rationaliste, avec cette attention particulière apportée au climat, au matériau, au site, comme nous le montre une de ses dernières œuvres : le château du Doux à Altillac, en Corrèze.

\section{L'enseignement, une vocation pour Jean-Louis Pascal}

3 «En 1872, alors que Questel ${ }^{6}$ se retirait : “Qui désirez-vous pour me succéder ?” demandat-il à ses élèves. "Pascal" répondit l'atelier, unanime " ${ }^{7}$.

4 Très jeune, à 35 ans, Jean-Louis Pascal avait pris la tête de l'atelier de Charles Auguste Questel dont il avait été lui-même élève (ill. 1).

\section{1 : L'atelier de Pascal en 1903}

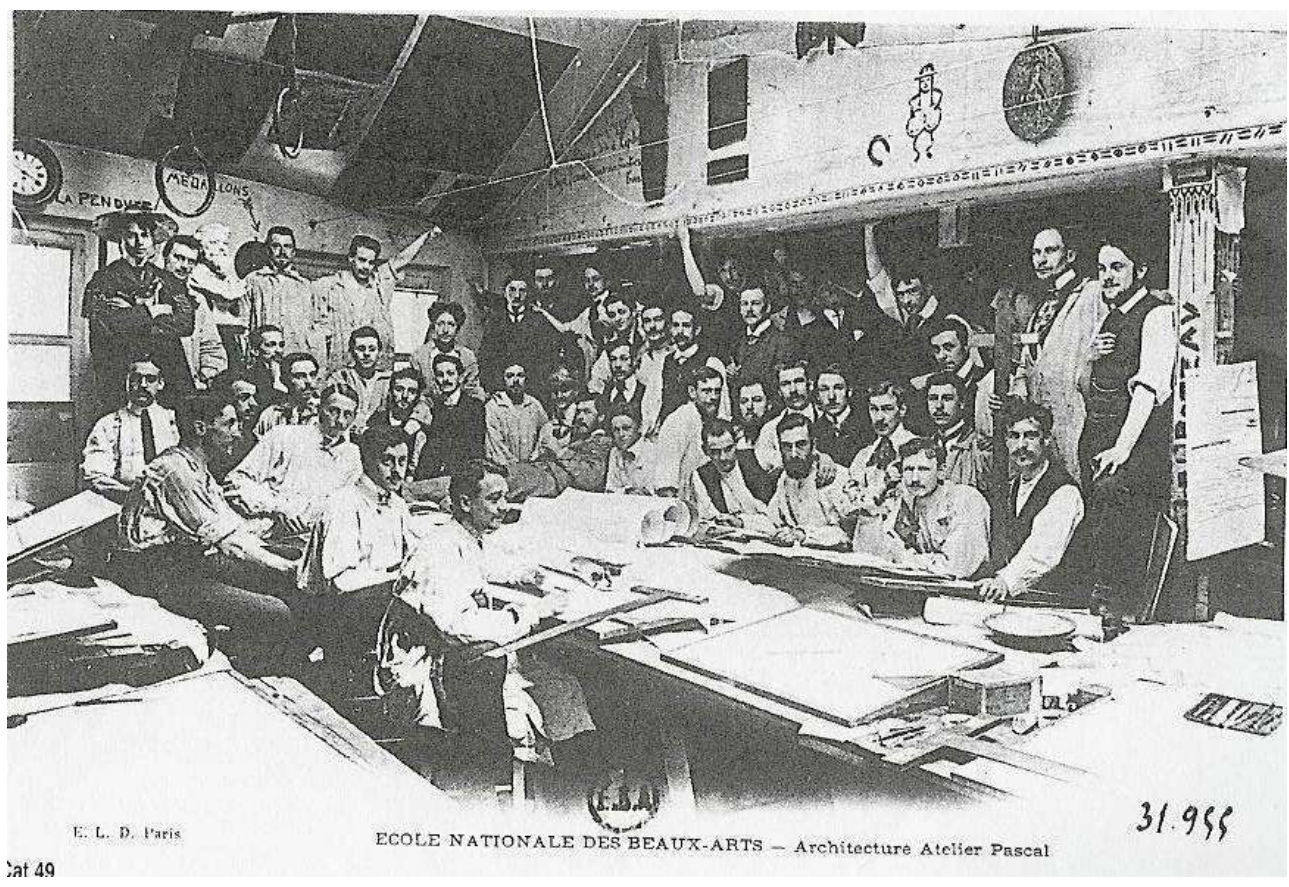

Annie Jacques, La carrière de l'architecte au XIXe siècle, Paris, R.M.N., 1986, 72 p., p. 27

Selon l'historien Michel Gallet ${ }^{8}$, dont le père André Gallet (1880-1950) fut l'élève de JeanLouis Pascal et le bibliothécaire d'atelier entre 1901 et 1908, « Pascal était vénéré, écouté, respecté ». L'homme était « très bon, très généreux » ${ }^{9}$ et " a exercé une sorte de charisme pédagogique ». De très nombreux témoignages d'élèves vont dans ce sens; Édouard Eugène Arnaud (1864-1943), architecte D.P.L.G., ingénieur des Arts et Manufactures, ancien élève de Jean-Louis Pascal, lui écrit en 1912: «Je puis vous dire que de tous les maîtres que j'ai eus, vous êtes celui qui a eu et qui aura dans ma vie la plus grande influence, celui dont je désire l'estime $»^{10}$. 
Il y avait trois grands ateliers à l'époque : celui de Jules André, celui de Gaston Fernand Redon (1853-1921) ${ }^{11}$ et celui de Pascal, «trois ateliers fortement marqués par l'éclectisme "Beaux-Arts" où se forma le style officiel de la Troisième République $»^{12}$. En 1872, Pascal prend la tête de l'atelier situé au $n^{\circ} 20$ de la rue Mazarine, pas très loin de la maison où Jean-François Champollion (1790-1832) avait vécu, quelques cinquante ans auparavant ${ }^{13}$. Jean-Louis Pascal y enseignait la composition classique, dont Henri-Paul Nénot fut certainement le représentant le plus brillant, mais son enseignement se caractérisait aussi par une grande ouverture d'esprit :

« Son enseignement était caractérisé par un éclectisme étonnant dans cet esprit si classique [...] Corrigeant un jour l'esquisse d'un tout jeune aspirant qui lui présentait un kiosque à musique chinois, il lui disait : "Faites du chinois, si vous y tenez, mais faites du bon chinois !" Il dirigeait ainsi ses élèves dans la voie de la raison tout en laissant se développer librement leur personnalité $\aleph^{14}$.

7 Henri Sauvage, qui avait été son élève, s'insurgea fréquemment contre l'enseignement académique qui était prodigué aux Beaux-Arts : il partit de l'École avant l'obtention de son diplôme ; «mais son œuvre et celle de nombre de ses camarades d'ateliers démontre que Pascal avait su leur transmettre - malgré un enseignement fondé sur l'étude historique de l'architecture - les bases indispensables pour dépasser cet académisme : parmi les jeunes contestataires qui exposèrent chez Le Barc de Boutteville ${ }^{15}$ en 1896, la majorité sortait de l'atelier de Pascal : André Jules Collin (1875-1966), Gabriel Gilbert Guillemonat (1866-?), Ernest Marie Herscher (1870-1939), Henri Sauvage ${ }^{16}$.

8 En $1897^{17}$, une grande fête avait été organisée dans les salons Marguery pour fêter le jubilé de Pascal: ses vingt-cinq années d'enseignement. Cent cinquante-deux convives y participaient dont Henri Roujon, directeur des beaux-arts, le graveur Jules Clément Chaplain (1839-1909), les architectes Édouard Jules Corroyer (1835-1904), Henri-Paul Nénot, Joseph Émile Auguste Vaudremer (1829-1914), membres de l'Institut, Leclerc, Gustave Raulin, le sculpteur Gustave Germain. Les amis et élèves de Pascal avaient répondu nombreux à l'appel du comité, qui, sous la direction de l'infatigable Félix Debat (1867- ?), massier sortant, préparait depuis plusieurs mois cette fête avec un dévouement témoignant de l'affection profonde que tous portaient à leur professeur. À cette occasion, Jules Chaplain grava une médaille ${ }^{18}$ : sur la face de la plaquette, se profile la fine et belle tête de Jean-Louis Pascal. Sur le revers, un chêne vigoureux, au tronc duquel est attaché un cartouche portant les dates : 1872-1897, occupe la partie gauche. De ses puissantes racines sortent de nombreux et vigoureux rejetons, allusion à ses nombreux élèves, «abrités par son ombre et nourris de sa sève ». Au loin s'étend la plaine, la campagne de Rome avec ses aqueducs. À l'horizon se profile le dôme de Saint-Pierre. Deux personnages, deux des principaux rejetons de l'arbre roi, saluent la ville sainte ${ }^{19}$. Trois élèves de Pascal étaient alors pensionnaires à la Villa Médicis : Ernest Alfred Henri Recoura (1864-1939), L. Pills et Eugène Duquesne, tout juste lauréat du grand prix d'architecture. La médaille, enchâssée dans un cartouche de marbre réalisé par le sculpteur Gustave Germain, fut offerte au patron par Paul Héneux, le plus ancien des élèves de l'atelier Questel au moment où l'atelier passa dans les mains de Pascal. Cinquante-huit élèves ou amis furent d'autre part invités à illustrer les feuillets d'un livre-album ${ }^{20}$ (ill. 2). 
III. 2 : Livre-album offert à Pascal en 1897 par ses élèves et amis à l'occasion du jubilé de son enseignement en tant que chef d'atelier libre à l'Ecole des Beaux-Arts

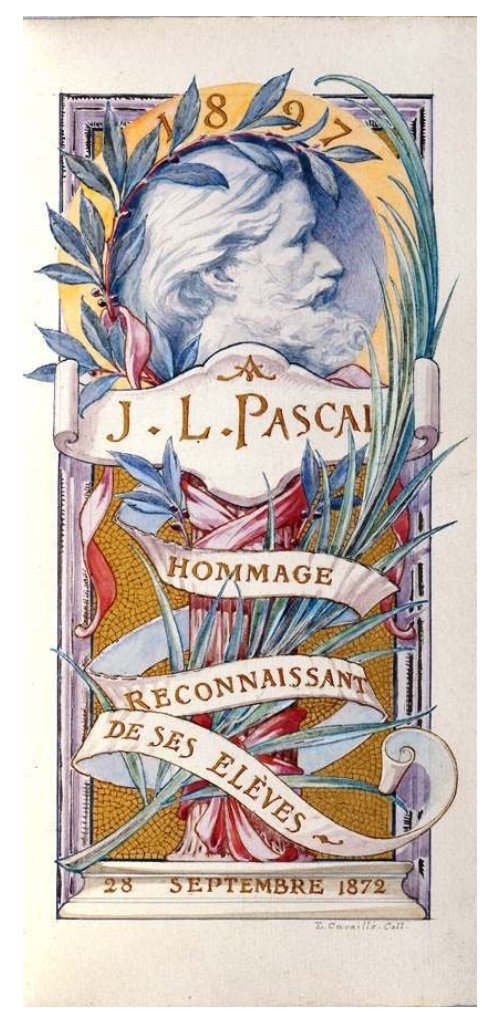

Page de garde. 1897

B.n.F., département des estampes, Pd 122, réserve, in $8^{\circ}$.

9 Chaque feuillet est un dessin original signé par un groupe d'élèves de Pascal (ill. 3 et 4); le livre contient ainsi la liste de tous ses élèves et celle de tous les anciens camarades souscripteurs. 
III. 3 : Page du livre-album offert à Pascal par ses élèves à l'occasion de son jubilé

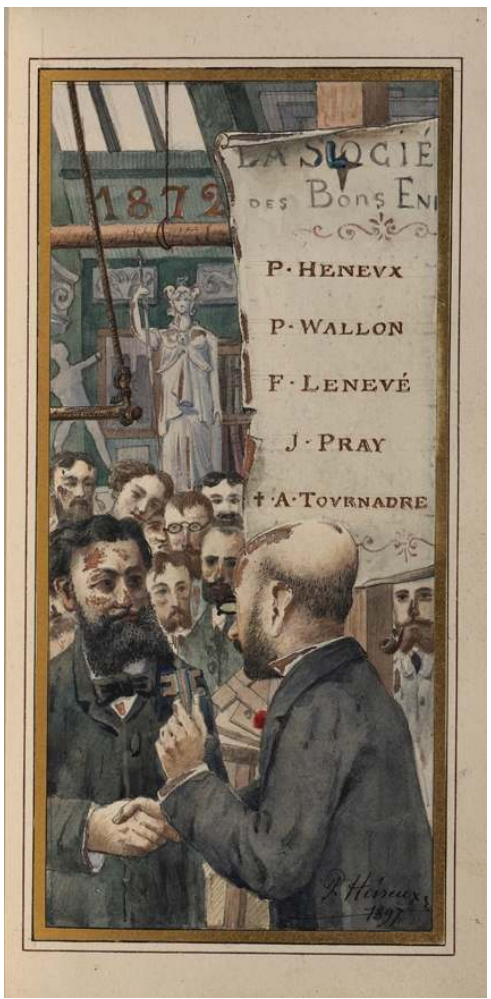

Signé : Paul Héneux. 1897

B.n.F., département des estampes, Pd 122, réserve, in $8^{\circ}$ 


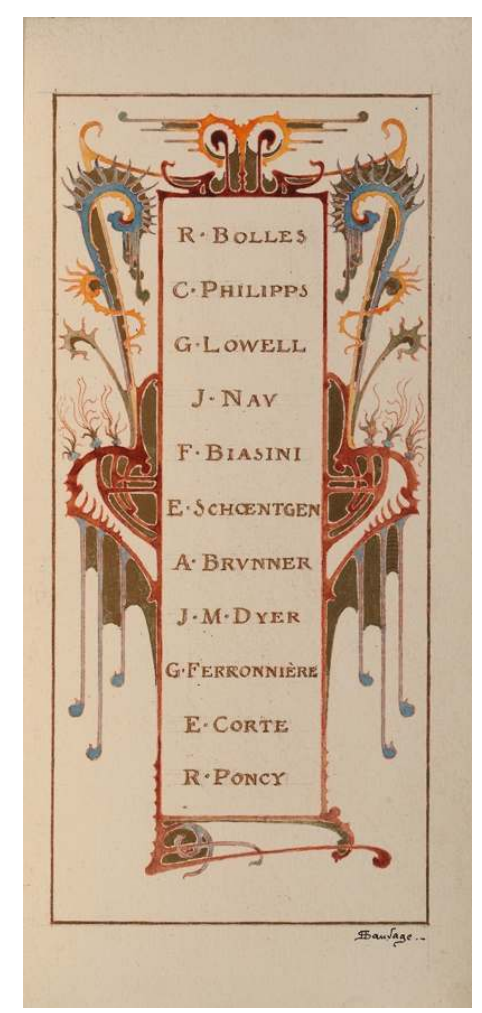

Signé : Henri Sauvage. 1897

B.n.F., département des estampes, Pd 122, réserve, in $8^{\circ}$.

L'album est luxueusement réalisé : la couverture est en maroquin, avec branches de lierre en bronze appliquées, les pages de garde sont en soie spécialement tissée à Lyon, la pâte du papier porte le monogramme de Pascal. Une association amicale d'anciens élèves de l'atelier Blouet-Gilbert-Questel-Pascal, est créée en 1893; quatre ans plus tard, elle compte trois cents membres ${ }^{21}$.

Pascal s'est toujours engagé sur la question de l'enseignement à l'École des beaux-arts. Il y consacre un grand article dans la R.G.A.T.P. en $1874^{22}$ où il s'insurge contre le décret de 1863 qui avait tenté de soustraire l'École à l'influence de l'Académie et d'encadrer sa liberté. Pour lui, la liberté de l'artiste ne peut être limitée ; or la création d'ateliers spéciaux et gratuits résultant de cette réforme, avait entraîné la fermeture des ateliers libres payants, réduisant, pour les élèves, la possibilité du choix de leur professeur. Le 24 mai 1898, le conseil supérieur d'enseignement de l'École des beaux-arts dont Pascal fait partie, statue sur le remplacement de Gustave Moreau (1826-1898) comme chef d'atelier de peinture, un artiste dont Pascal avait une haute opinion : «Un artiste bien intéressant à mon goût, bien original, bien personnel $\aleph^{23}$. Un seul candidat se présente: Thirion; Pascal y voit la preuve de l'inutilité des ateliers officiels ${ }^{24}$.

Dès la Villa Médicis, Pascal avait œuvré pour les pensionnaires : "Les lauréats du concours de 1866 étaient partis de Paris un peu tardivement [...]. C'étaient des fantaisistes. En leur nom, le 18 janvier 1867, l'architecte Pascal écrivait au directeur [...] Peu fortunés, ils ne voulaient point perdre le bénéfice du premier mois de leur pension et, gentiment, ils s'en ouvraient à Robert-Fleury ${ }^{25}$ en lui demandant quelques jours de sursis jusqu'au 2 ou 3 février. La lettre était déférente au possible. Elle ne toucha pas Robert- 
Fleury qui, en marge de la prose de Pascal, écrivit : "Répondu le 22 - pas accordé." »². Il faut rappeler que la pension accordée aux élèves à Rome était extrêmement modique; Paul Baudry, grand prix en 1850, s'en plaignait déjà, seize ans auparavant : dans une lettre à ses parents, il écrit : «La pension qu'on nous alloue est beaucoup trop chétive pour nos besoins: le bois, la chandelle, les ports de lettres, les domestiques de chambre, les cuisinières conspirent pour nous arracher lopin par lopin les cent francs que nous donne le directeur au commencement de chaque mois $»^{27}$. Et puis il $\mathrm{y}$ avait le problème des voyages : « Hébert ${ }^{28}$, ayant obtenu pour les pensionnaires ce qu'ils désiraient, demanda à son tour qu'on l'armât contre l'abus du voyage à Paris, lequel lui paraissait "en désaccord avec l'esprit et la lettre du règlement nouveau" [...] et pourtant les pensionnaires obtinrent de lui qu'il ne se montrât pas impitoyable, au moins pour cette année qui était celle de la seconde exposition universelle [1867]. Ingres était mort au mois de janvier et son exposition posthume allait s'ouvrir à l'École des beaux-arts $»^{29}$. Une pétition fut signée notamment par Pascal à Rome le 23 mars 1867 : «À la suite de la pétition, le surintendant Nieuwerkerke agréa les vœux ainsi exprimés, à titre exceptionnel et à condition que les pensionnaires rempliraient toutes leurs obligations [...] Bientôt, profitant de l'autorisation de Nieuwerkerke, les pensionnaires abandonnaient la Villa Médicis. La plupart partaient pour Paris. Noguet et Gerhardt étaient en Grèce, Laguillermie voyageait en Orient et Maillard ${ }^{30}$ se fixait à Venise. Pascal, lui, allait à Salerne étudier la cathédrale $»^{31}$. Le graveur Laguillermie proposa d'ailleurs pour sa restauration de quatrième année la gravure d'une planche d'après le tableau Les Lances du musée du Prado à Madrid. La commission chargée de juger les envois des pensionnaires de Rome jugea son choix inapproprié :

«Le grand prix de gravure a été institué pour faciliter l'instruction des jeunes artistes et les initier à la connaissance des grands maîtres de l'Italie. Vélasquez n'offre pas les éléments nécessaires au but qu'on s'est proposé [...] M. Laguillermie semblait annoncer un talent distingué. Ses heureuses dispositions et son intelligence nous en donnaient l'espérance. Malheureusement il s'est égaré par une mauvaise direction dans ses études et surtout par des voyages inutiles qui lui ont fait perdre un temps précieux $»^{32}$.

13 Et, jugeant la même année la restauration de la Villa Madame ${ }^{33}$ par Henri Bénard, la commission ajoutait :

«Ce n'est que lorsqu'on veut voir autre chose qu'un temps d'études sereines passé à la Villa Médicis qu'on songe à se plaindre de la rareté des sujets nouveaux. Si le pensionnaire se préoccupe trop de son retour et de l'accueil qui l'attend à son retour à Paris il cherchera évidemment un effet d'exposition répondant au goût présent et à la mode, alors Paris réagira sur Rome ce qui est juste le contraire de ce qu'a voulu l'État en créant des écoles à l'étranger " ${ }^{34}$.

Pascal écrivait en 1874 :

" Nous n'attachons d'ailleurs qu'une médiocre importance aux modifications apportées au règlement intérieur de l'Académie de France à Rome, tant qu'elles n'aliéneront pas la liberté du pensionnaire. Les envois ne forment qu'une partie de leurs travaux ; il est bon que tous soient astreints à certaines études qui constituent le fond commun ; hors cela, qu'ils aillent où bon leur semble, au risque de s'égarer. Les écarts les plus imprévus ne sont point à craindre chez des travailleurs ayant donné les garanties qu'ils ont fournies ${ }^{35}$.

15 En 1890, alors qu'il a été nommé à l'Institut ${ }^{36}$, il reverra sa position ${ }^{37}$ dans un rapport qui expose que, sur les voyages hors de l'Italie, la Grèce et la Sicile, il faut garder le règlement et laisser au directeur de l'Académie de France à Rome le soin de donner comme par le passé des autorisations exceptionnelles. L'autorisation de restaurer un édifice autre 
qu'antique en quatrième année, c'est-à-dire un édifice de la Renaissance ou du Moyen Âge, est d'autre part refusée car ces monuments « ne sont pas assez ruinés pour qu'on puisse trouver à les imaginer à nouveau ».

L'année suivante ${ }^{38}$ le règlement est modifié : les pensionnaires sont tenus de rester pendant la première année de leur pension à Rome et en Italie. Les pensionnaires peintres pourront, avec l'autorisation du directeur de l'Académie, exécuter hors de l'Italie, la France exceptée, la copie qu'ils sont tenus de faire au cours de la troisième année de leur pension. Le même règlement était appliqué pour les élèves architectes, pour la restauration d'un édifice ou d'un ensemble d'édifices antiques: «Les pensionnaires d'aujourd'hui subissent d'autres influences. Souvent Rome leur semble un exil; ils regrettent Paris, sa vie brûlante, son indépendance, son art même. On ne va à Rome qu'à regret, on en revient au plus vite $»^{39}$.

\section{La lutte contre l'internationalisation des styles}

\section{Un constat à l'exposition universelle de Paris (1900)}

17 Pascal rédige régulièrement les comptes rendus du Salon pour la section d'architecture, en tant que membre du jury. Il sera membre du jury des récompenses pour la section d'architecture de l'exposition universelle de $1889^{40}$ (il obtiendra une médaille à cette occasion) et rapporteur ${ }^{41} \mathrm{du}$ jury de la section d'architecture de l'exposition universelle de 1900: "Aucune exposition universelle n'avait encore fourni une place pareille à l'élément architectural $\aleph^{42}$. On y voit Pascal s'insurger contre l'internationalisation des goûts ${ }^{43}$ et le manque d'imagination constaté dans les productions françaises. Charles Garnier dénonçait déjà ce phénomène en 1887, lorsque, chargé de faire un discours lors de la séance publique solennelle de l'Institut, il s'insurgeait contre " les mêmes formules banales, les mêmes clichés de composition [qui] se répandent dans chaque nation [...] les mêmes matériaux de construction qui s'emploient dans toutes les cités, en retirant ainsi à l'architecture son caractère primordial et rationnel $»^{44}$. Selon Jean-Louis Pascal, la constitution définitive de l'unité française avait fait disparaître les distinctions d'écoles, bourguignonne, picarde, champenoise, normande, alors que l'Allemagne et l'Italie, deux pays qui s'étaient constitués de manière analogue, avaient gardé des centres d'art bien vivants. Traversant l'Allemagne, alors qu'il se rend à Vienne en chemin de fer pour l'exposition universelle de 1873, il écrit:«Les stations passent, variées, coquettes, typiques, non point atteintes comme chez nous de la monotonie administrative, mais $s^{\prime}$ harmonisant par leur composition et leurs matériaux, avec leur entourage même $»^{45}$. On en trouve un écho ${ }^{46}$, quelques trente ans plus tard, dans les propos de Henri Cazalis, alias Jean Lahor (1840-1909), membre de la Fédération régionaliste française créee par Jean Charles-Brun (1870-1946) en 1900, et fondateur de la Société d'Art populaire et d'Hygiène ${ }^{47}$ : «J'aimerais [...] que la maison du paysan, [...] on la laissa bretonne en Bretagne, savoyarde en Savoie, provençale en Provence, normande en Normandie, flamande dans les Flandres, [...] parce que la variété dans l'unité est un principe d'art vrai toujours, l'uniformité suant l'ennui »48.

Pascal craignait ainsi que l'art russe ne perdît son originalité : «Combien nous aimerions à voir les artistes russes, s'inspirant de leur patriotisme même, de leur génie propre, de leurs traditions, de leur race, rester fidèles à leur passé $! »^{49}$. Mais c'est peut-être pour le 
Japon, dont l'exposition à Vienne en 1873 l'avait émerveillé, que l'architecte craignait le plus cette uniformisation des styles :

«Ce dernier pays, notamment, mériterait au moins une mention pour la gracieuse et étrange imagination et le brillant sentiment de couleur qu'il déploie dans ses étoffes, ses vases, ses objets d'usage journalier, dans ses petites maisons de bois et de papier [...] son art indigène, son architecture suivront aussi les influences de l'Occident, comme son armée, sa législation et le reste. Puisse-t-il ne pas perdre à ce frottement toute la saveur de son originalité, toutes les qualités charmantes qui indiquent une race éminemment artiste! Déjà le costume est attaqué et, avec lui, l'industrie de la soie. Il est à craindre que notre civilisation, pénétrant tout d'une pièce, n'étale sa triste uniformité sur les formes et sur le fond, et ne transforme en une colonie à chapeaux de soie et à faux-cols ce riant pays, de mœurs et de couleurs si particulières ${ }^{50}$.

Comme symbole de cette lutte, sans doute perdue d'avance, contre l'émergence d' " une architecture cosmopolite résultant de la suppression de l'espace, du temps et presque des latitudes $\aleph^{51}$, le jury de l'exposition de 1900 attribua une récompense exceptionnelle : un grand prix à un vieil artiste japonais ; Jean-Louis Pascal s'en explique :

" Cet entraînement pour l'art de l'Extrême-Orient était peut-être le dernier salut donné à des formes qui s'évanouissent malheureusement pour faire place aux aspirations de notre civilisation du vieux monde latin et grec, à l'influence anglosaxonne, aux formes déjà continuées dans le Nouveau Monde américain qui ne pouvait avoir d'autres traditions que les nôtres puisqu'elles proviennent de la même origine [...] La connaissance superficielle que l'archéologie, l'histoire, les voyages plus fréquents nous ont donnée de ces formes a bien retenti jusque chez nous en une influence trop de fois signalée pour qu'il vaille la peine de s'y étendre ici : le cosmopolitisme aura fait une œuvre inverse avant de couvrir tout de son manteau bigarré de pièces et de morceaux [...] Autrefois dans les étapes successives de notre art, l'unité caractérisée par les styles, les siècles, les noms des souverains se nuançait pourtant du petit caractère et de l'influence des provinces, sans parler des matériaux mis à la disposition des constructeurs dans le court rayon de leur action. Il serait bien puéril maintenant d'essayer d'enrayer le mouvement logique et inévitable qui promène son niveau égalitaire sur nos conceptions, y étalant la confusion et la similitude des formes, conséquence aussi de l'analogie des mœurs. Un pays de petite surface comme la France laissera de moins en moins se différencier ses constructions du Midi de celles du Nord ${ }^{52}$. Comment y parler de décentralisation en même temps que le développement des moyens de transport donne toutes facilités pour user partout des mêmes matériaux, quand la pénétration par l'image, par l'enseignement, par l'exemple traverse jusqu'aux frontières et aux océans ? Les civilisations de même origine sont condamnées à perdre leur particularisme pour se fusionner dans de plus grandes écoles, nationales au lieu de provinciales et plus encore : universelles au lieu de nationales [...]

Exceptionnellement, des tentatives spéciales, sous le nom d'art nouveau, de "modern style", de symbolisme, etc., ont tenté de traduire pour nous des tendances particularistes, signalétiques de génie national, et encore devons-nous dire qu'elles se ressemblaient en Belgique, en Autriche, en Angleterre, en France, comme filles d'une commune fantaisie.

Nous les enregistrons toutefois au passage dans un esprit de cordiale sympathie comme tous les efforts d'art qui peuvent faire vibrer nos souvenirs intimes au milieu de cette sorte de faillite aux tendances de rénovation du commencement du siècle [...] En France même, là où s'élaborent plus spécialement les longues études passionnément suivies, ne voyons-nous pas le plus noble, le plus grand de nos palais nouveaux ${ }^{53}$, œuvre digne à tous égards de la haute estime que nous lui avons témoignée, porter la trace d'une époque 
expirée et remonter à cent ans, pour accuser sur une composition récente, un style du passé devant caractériser l'art de $1900 ?{ }^{54}$.

Et de conclure par :

"Comme, à des époques lointaines, on a vu le style des maîtres du monde se répandre sur les rives de la Méditerranée, nos successeurs subiront peut-être, non sans surprise et sans regret, d'autres maîtres plus impérieux, la démocratie, le goût, la liberté des échanges, le nivellement des fortunes, l'expansion des voyages, la facilité des transports et l'ampleur des transactions, qui provoqueront la similitude et l'uniformité. De nouvelles recherches et de troublantes inventions supprimeront les frontières, renverseront nos petites barrières et nous amèneront aux fusions les plus inattendues, aussi éloignées des résultats actuels que ceux-ci le sont des divergences datant d'un siècle. L'art pourra y trouver une place agrandie $»^{55}$.

\section{La création des Écoles régionales d'architecture}

23 Bien qu'ayant contesté la création d'un " diplôme provincial » ${ }^{56}$ d'architecture, Pascal va prendre une part active dans la création des Écoles régionales d'architecture. Il écrit en 1904 à Stanislas Ferrand, directeur du journal le Bâtiment : « Vous m'avez fait l'honneur de m'adresser votre appréciation sur les écoles régionales d'architecture. C'est apparemment parce que vous savez que nous y avons pris, de grande bonne volonté, à quelques-uns qui ne pouvions guère y trouver que surcharge d'occupations, une part empressée». Il y développe un argumentaire destiné à convaincre Ferrand qui lui reprochait « d'avoir aussi mal employé son temps et ses courses à travers la France $»^{57}$ :

«Faisant la part de la différence entre une éducation scientifique [...] d'un très grand centre et de plus petits milieux moins suggestifs, il n'y avait pas un de nous, professeurs officiels ou libres [...], qui ne constatât que le commencement de nos concours, que d'abord l'admission à l'école, pussent aussi bien s'organiser sous le contrôle de la famille, entre la dix-septième et la vingtième année, à Toulouse ou à Lille, qu'à Paris. Et comme la longue fréquentation des jeunes gens [...] montrait qu'il serait bien désirable que de trop jeunes gens ne fussent pas lâchés à tort et à travers dans notre grande ville, nous fûmes frappés, touchés par ce désir de gens expérimentés de sauvegarder les générations à venir, en même temps que de faciliter aux pauvres le commencement d'études qui leur était littéralement impossible. [...] Nous passâmes, de parti pris, par-dessus [...] tous les si, les mais qui s'élevaient contre la création même sommaire, même insuffisante, de ces succursales de l'́école de Paris (sic) $\Perp^{58}$.

Et lorsque l'on reprochera à Pascal de susciter ainsi la disparition des architectures locales, l'architecte répondra que l'on ne peut lutter contre cette internationalisation des styles : «Est-ce qu'il va pouvoir exister des architectures locales? [...] Est-ce que les architectes formés à Marseille s'engageront à ne pas construire à Biarritz? Est-ce que ceux de nous dont la carrière est longue n'ont pas semé leurs œuvres de Dunkerque à Bayonne? Est-ce que les Américains qui viennent étudier à Paris se serviront de nos matériaux ? $»^{59}$.

Pascal sera toujours très assidu aux séances du conseil supérieur de l'enseignement de l'École des beaux-arts, comme il l'était à celles de l'Institut, étant passionné par tout ce qui touchait à l'enseignement de l'architecture, à Paris comme en province. À la suite de la discussion du budget de 1901 et conformément aux demandes réitérées de toutes les grandes sociétés provinciales d'architectes, le ministère de l'instruction publique et des beaux-arts se résolut à créer des Écoles régionales d'architecture. Une commission fut nommée pour faire des études préliminaires; elle comprenait: Léon Bonnat, Émile 
Vaudremer, Jean-Louis Pascal, Julien Guadet, Henri-Paul Nénot, Jean-Paul Laurens (1838-1921), Fernand Cormon (1845-1924), Jules Coutan, Hugues Valentino, Henri Roujon, Paul Richer, Lemonnier. Il y eut de nombreux débats au sein du conseil supérieur d'enseignement de l'École des beaux-arts. Pascal et Guadet réclamaient pour les élèves des Écoles régionales le droit de venir à Paris quand bon leur semblerait. Hugues Valentino y voyait un danger : celui de vider les Écoles régionales; ou alors il fallait imposer que les élèves provinciaux aient déjà fait leurs études de seconde classe. Lemonnier craignait que les Écoles régionales ne soient abandonnées par les élèves les plus brillants et ne deviennent des écoles de préparation à l'École des beaux-arts de Paris. Le docteur Paul Richer voulait donner aux Écoles régionales d'architecture une autonomie complète ${ }^{60}$. L'enquête de la commission terminée, Julien Guadet présenta un rapport. Le ministre approuva les propositions de la commission, fit signer les décrets ${ }^{61}$ relatifs à la création des Écoles régionales d'architecture et insérer au Journal officiel en même temps que ces décrets, le texte du rapport de Guadet, considéré dès lors comme le commentaire officiel ${ }^{62}$ :

- Les Écoles régionales, comme l'École des beaux-arts, devaient pouvoir conférer le diplôme d'architecte, dès lors il devait en résulter l'unité des études dans ces différentes écoles.

- Le candidat ayant passé avec succès les épreuves d'admission, quelle que soit l'école où il les aurait subies serait de droit et de fait admis, non seulement à telle ou telle école, mais à celle où il lui plairait de s'inscrire.

28 - Plus tard, s'il voulait transporter ailleurs sa résidence, les valeurs acquises par lui le suivraient là où il se transporterait. Celui qui, ayant commencé ses études à Paris voudrait retourner dans sa région, pourrait y continuer ses études et vice-versa.

Pourtant, le ministre dut prendre une disposition différente; il était évident que le flux province-Paris serait le plus important; il décida donc de statuer au cas par cas. Il faudrait des circonstances imprévues ou exceptionnelles pour autoriser un élève à venir à Paris.

L'avis du conseil supérieur d'enseignement fut le maintien de ce statu quo pour les élèves de seconde classe et l'autorisation sans condition pour ceux de première classe.

31 Ce moyen terme fut choisi, avec le risque de voir les Écoles régionales devenir des écoles préparatoires pour l'École des beaux-arts.

32 En 1909, une pétition, demandant la modification de l'organisation des Écoles régionales d'architecture, déposée par les élèves des Beaux-arts de Paris, entraîne la constitution d'une commission ${ }^{63}$ chargée d'examiner une proposition relative aux concours d'admission d'architecture. En effet, certaines épreuves pour le concours d'admission ou le passage de la seconde à la première classe, offraient moins de difficultés en province qu'à Paris. Les élèves des Écoles régionales entraient plus vite en seconde, puis en première classe, gagnant ainsi du temps sur les élèves de Paris. Étaient admis à passer en seconde classe à Paris les quarante-cinq candidats français ayant obtenu les sommes de points les plus élevées, en province étaient admis ceux ayant obtenu au moins le nombre de points attribué à l'élève classé dernier à Paris. Les élèves de l'École des beaux-arts proposaient, soit une centralisation complète de l'enseignement avec les mêmes jurys, les mêmes conditions de surveillance, une intégrité absolue du concours et un diplôme unique, soit, et c'était la solution qu'ils préféraient, une décentralisation absolue avec autonomie complète : administration, budget, règlements, concours, jurys particuliers et 
diplôme spécial pour chaque école régionale. Ils demandaient "non pas des demimesures et des temporisations chères aux craintifs et aux impuissants, mais des solutions ». Leur pétition est transmise le 15 janvier 1910 à la commission et leurs plaintes contre les Écoles régionales sont rejetées.

Jean-Louis Pascal, proposa de remplacer par un examen le concours d'entrée à l'École des beaux-arts ; Pascal s'insurgeait depuis longtemps contre le numerus clausus prévalant à l'entrée à l'École (seules l'École de Saint-Cyr et l'École polytechnique recrutant suivant les nécessités des grands services publics et de l'armée étaient, selon lui, fondées à le pratiquer). Henri-Paul Nénot ne partageait pas le point de vue de Pascal ; pour lui, l'École des beaux-arts devait rester un établissement d'enseignement supérieur. La proposition de Pascal fut rejetée ${ }^{64}$.

En 1913, le conseil supérieur charge une nouvelle commission de réétudier le problème du passage des Écoles régionales à l'École des beaux-arts. En effet les Écoles de province ont été décapitées. Désormais, les élèves de première classe de province, passés à Paris, sont obligés de participer en province à trois des concours de projets rendus, à moins qu'ils n'aient été admis en loge pour le concours de Rome ${ }^{65}$.

Quelques années auparavant, en 1899, Jean-Louis Pascal avait eu le grand honneur d'être choisi comme président du jury du concours destiné à choisir l'architecte de la nouvelle université de Berkeley en Californie ${ }^{66}$. Pascal en profita pour voyager aux États-Unis durant deux mois et demi, multipliant les contacts avec d'anciens élèves installés outreAtlantique. C'est peut-être lors de ces dîners amicaux que lui sont présentés $\mathrm{M}$. et $\mathrm{M}^{\mathrm{me}}$ Bertin; G. Bertin est un parisien, écrivain amateur, chercheur de curiosités et de biographies intéressantes; sa femme est américaine. Ils se sont pris de passion pour un coin vallonné de Corrèze : Altillac, et ils demandent à Pascal de leur y construire un château.

\section{Une œuvre régionaliste : le château du Doux à Altillac, près de Beaulieu-sur-Ménoire (Corrèze)}

C'est près de Beaulieu-sur-Ménoire à Altillac en Corrèze ${ }^{67}$ que les Bertin, demandèrent à Jean-Louis Pascal ${ }^{68}$ de leur construire une belle demeure sur un terrain dominant la vallée de la Dordogne, en lieu et place d'une ancienne habitation familiale. Pascal aima construire ce château du Doux ${ }^{69}$ (ill. 5), qui empruntait son nom au ruisseau qui y serpente, pour des clients qui devinrent de véritables amis ; écrivant à Louise Garnier, de Corrèze, "au milieu des sonneries de leur trentaine de vaches", il dit "prendre des satisfactions à des insistances amicales de la part de clients qui [lui] ont donné les plus douces jouissances à leur édifier en montagne un grand château et trente-six choses avec ${ }^{70}$. " 


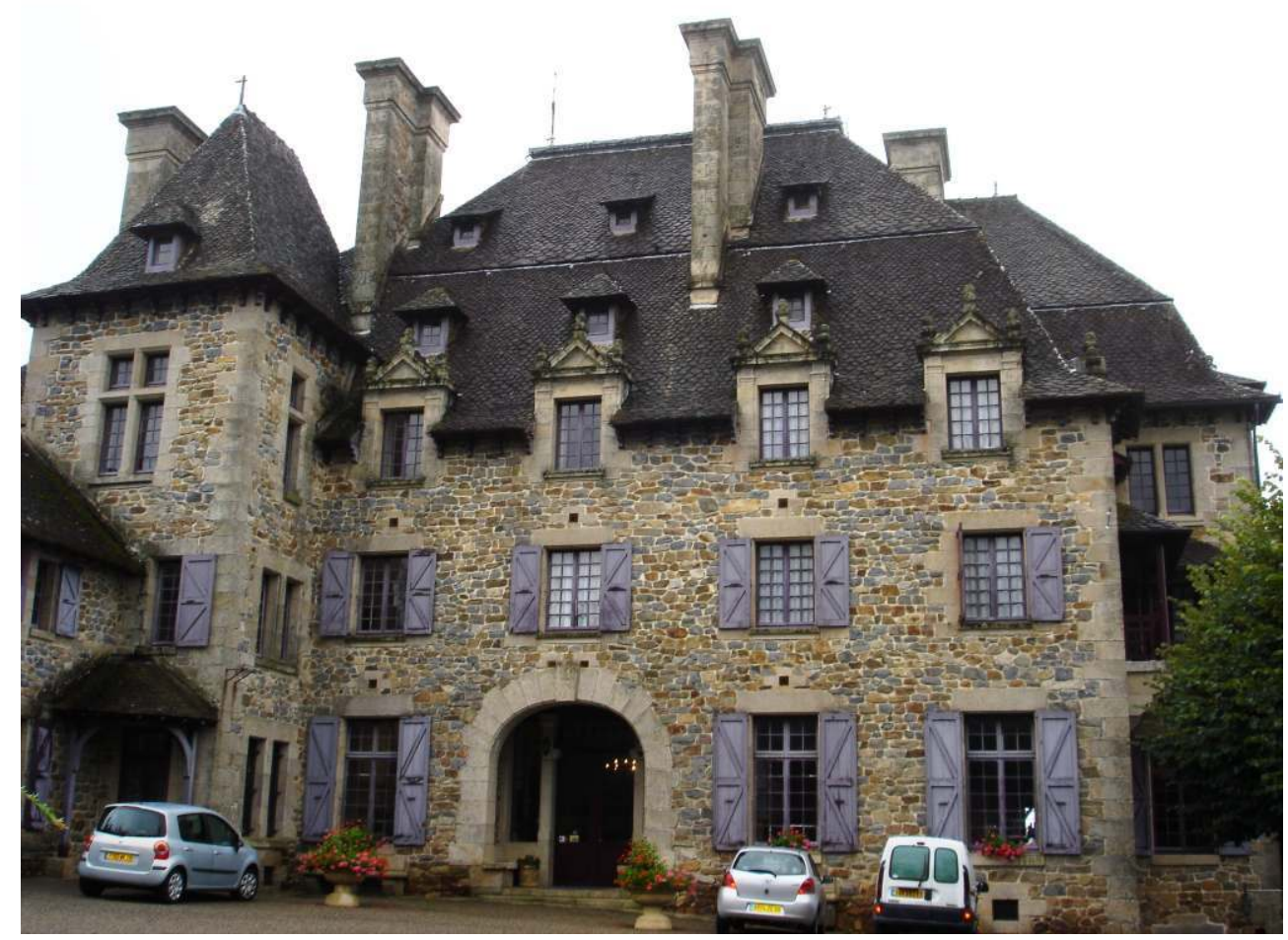

Porche d'entrée et façade nord. Architecte Jean-Louis Pascal. 1908

$\mathrm{Cl}$. auteur.

De larges cultures de céréales transformées en belles prairies au milieu de l'ensemble des bois de châtaigniers qui entourent le domaine, avaient permis de réaliser le vœu des propriétaires d'y installer une trentaine de bêtes à cornes : « On leur a donné un asile de caractère très local, tout à fait vers le sommet des pentes. L'usage est ici de choisir un terrain incliné et d'y établir, au-dessus du rez-de-chaussée destiné à l'étable, [...], sous un comble énorme, un grenier pour le fourrage ${ }^{71}$. Pascal créa d'abord, avec l'aide d'un artisan de Limoges, Mabille, tout un réseau de canalisations destiné à capter l'eau répartie abondamment dans la propriété. De grandes galeries furent créées dans le sol granitique et des puits d'accès furent aménagés. Un réservoir fut enfoui sous terre à mi-hauteur pour atténuer l'excès de pression qu'un niveau trop élevé aurait constitué. À l'ouest, Pascal installa avec l'aide de Deny, créateur de jardins, un ample potager qui s'étageait en pente douce sous une aile du château. Des bassins y étaient réservés pour mettre à température ambiante l'eau de l'arrosage. Pascal clôtura le domaine :

«En suivant les mouvements du terrain à partir d'un motif d'entrée, cherché et réalisé quelque peu brutal, des murs dévalèrent le long du potager, formant pour les fruits des appuis d'espaliers précieux, tournèrent en bas, en contact avec le bois, dans toute la longueur de la partie ouest, et plus loin, transformés en grillages au midi, revinrent vers les terrasses [...] pour rejoindre une partie ovale, face à l'est, composée de montants rustiques en pierre brute avec grilles et grillages dans l'intervalle. [...] Le visiteur, passé le grand portail, jouit d'une admirable vue par la terrasse dégagée sur la droite, coupée de quelques vieux noyers, et limitée à gauche par de la verdure, et une magnifique haie d'hortensias bleus ${ }^{72}$, derrière laquelle on a tout le bâtiment de dépendances. ${ }^{73}$ 
Au milieu de celui-ci, une large cour de pansage était, à l'origine, destinée à séparer les chevaux et les équipages des maîtres, de ceux des étrangers. Par la suite, elle fut affectée au service des automobiles. Les bâtiments arrière de ces dépendances contenaient une petite vacherie (étables et laiterie), des ateliers (selleries, boulangerie, atelier de photographie) et le premier étage, desservi par deux escaliers rustiques, était occupé par les domestiques et jardiniers, c'est-à-dire le personnel extérieur au service de la maison. Ce bâtiment est tout à fait isolé de la grande habitation, laquelle se compose d'un gros bloc avec façade principale au midi (ill. 6), une façade moins importante à l'ouest (ill. 6), la façade au nord (ill. 5) avec une entrée large et basse où, de l'avis de Pascal, il manquait un balcon de fer (un porche permet d'attendre à couvert sur des bancs de granit) ; à gauche, vers l'est, séparée de la façade nord par une tour carrée surmontée d'un toit à forte pente, mais décalée par rapport à elle, un bâtiment de communs avec entrée de service, cave, salle des gens, buanderie, cuisine communiquant par un office avec la salle à manger de la grande demeure.

\section{6 : Château du Doux à Altillac (Corrèze)}

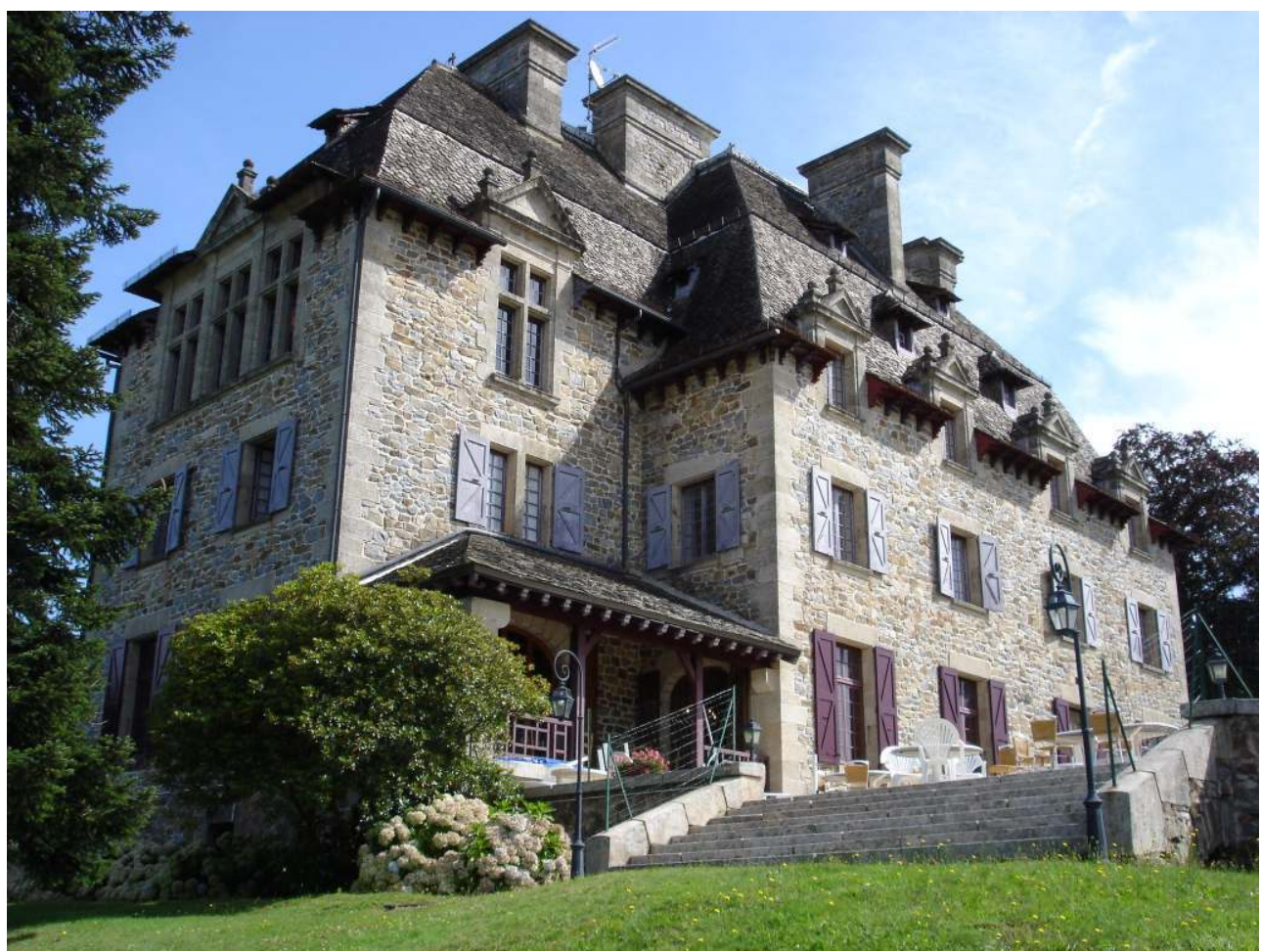

Façades ouest et sud. Architecte Jean-Louis Pascal. 1908. Façades ouest (avec les fenêtres de la bibliothèque au $2^{\mathrm{e}}$ étage) et sud : on distingue le petit abri dont le toit recèle un voligeage

Cl. auteur.

Toute cette partie, plus basse que l'édifice principal, est surmontée d'un étage d'habitation pour les femmes de chambre, domestiques et cuisinières employées au château. La façade est est de ce fait moins lisible (ill. 7). 
III. 7 : Château du Doux à Altillac (Corrèze)

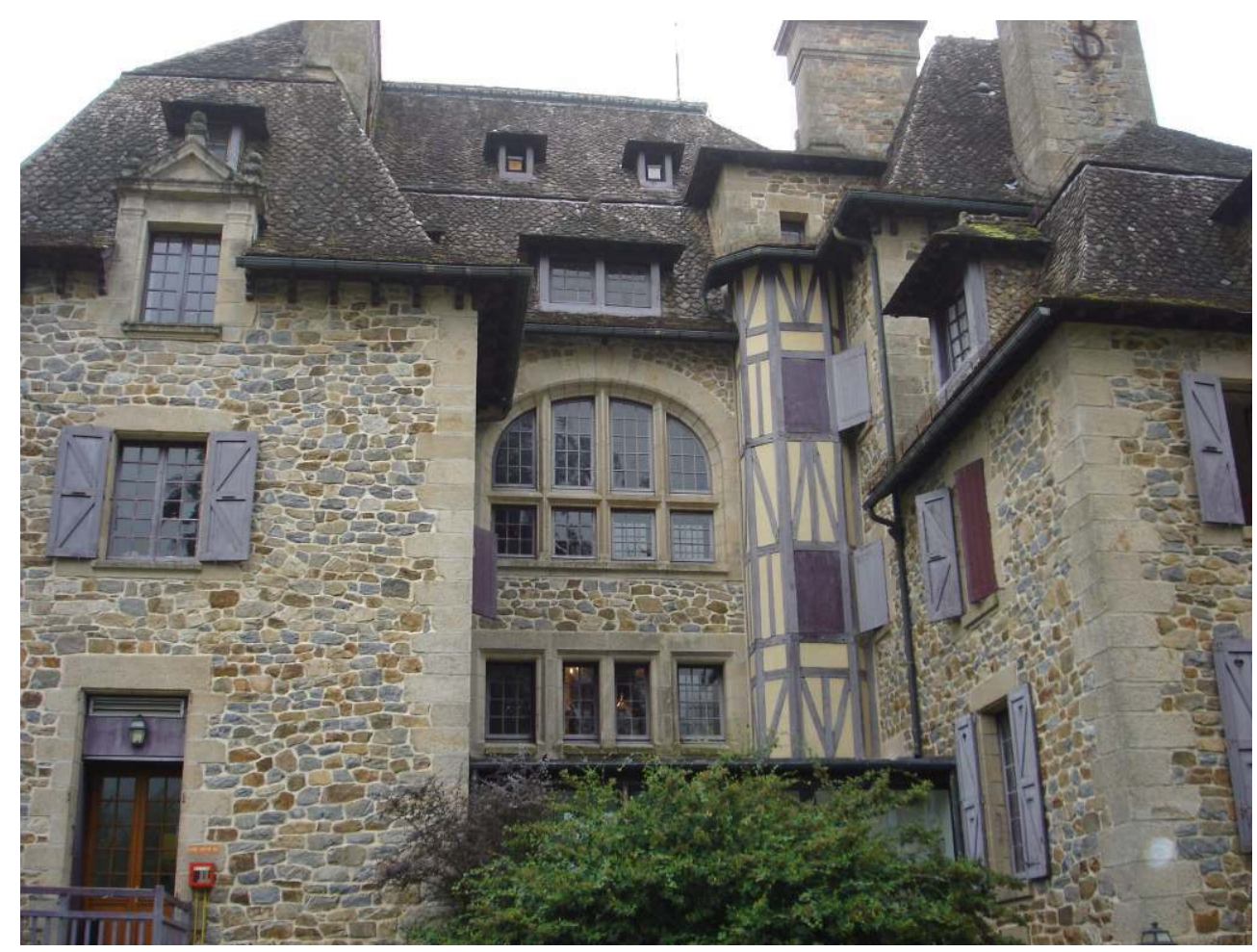

Façade est. Architecte Jean-Louis Pascal. 1908. Façade est avec sa tourelle en pans de bois et la silhouette pittoresque des toits. On distingue le « B » initiale du nom du propriétaire Bertin sur les cheminées

Cl. auteur.

40 Pascal y utilise ponctuellement l'architecture de pans de bois qui en tant de régions de France avait survécu jusqu'au XVIII ${ }^{e}$ siècle et était désormais réservée aux communs et aux tourelles contenant l'escalier ou les cabinets de toilette.

41 L'épouse de G. Bertin ne profitera malheureusement pas longtemps de cette maison puisqu'elle décède en mars 1910, laissant un mari désemparé; Pascal la définissait comme « une femme exquise [...] une parfaite créature de dévouement intelligent.»; " C'est pour ce couple [raconte Pascal à Louise Garnier] que j'ai fait en Corrèze un château avec grandes terrasses, dépendances importantes, routes, canalisations, etc, une œuvre de prédilection dans ma carrière $»^{74}$.

42 Louis Hautecoeur voyait dans la présence des grandes lucarnes sculptées dans les hautes toitures - tendance de nombre de châteaux prétendant rappeler ceux des bords de la Loire -, associées à l'usage de matériaux du pays, la marque de l'éclectisme de Pascal ; il semble que Pascal ait voulu conférer à son architecture un caractère typiquement régional car on retrouve ces lucarnes dans plusieurs constructions de la région; la forme si particulière du toit ainsi que l'usage de dalles schisteuses (ill. 8) pour le recouvrir sont tout à fait typiques de l'architecture locale; le poids des dalles obligeait les artisans du coin à employer une méthode spéciale de fixation à l'aide de chevilles de bois enfoncées dans le gros voligeage et dépassant par en-dessous; Pascal en fera l'essai pour un abri situé sur la façade sud, entre la salle à manger et le salon, sorte de petite terrasse en plein air (ill. 6 et 9) : 
"Quelque chose domine à l'extérieur cette composition compliquée, traduisant cependant la conception du plan ; c'est l'énorme hauteur des toitures avec cette forme presque arrondie, que donne la brisure des fermes, laquelle est extrêmement fréquente dans la contrée et s'y associe parfaitement avec les rondeurs et les grâces souples d'un paysage noble mais sans sévérité. Le programme qu'on m'avait donné était de faire une maison qui fût bien du pays ; je fis de mon mieux pour qu'elle fût bien aussi dans le paysage ; et on n'hésita pas à m'inviter à emprunter aux vieilles habitudes locales la couverture en pierre schisteuse par dalles de trois à quatre centimètres d'épaisseur, de grandes dimensions, chargeant furieusement la charpente et réclamant une expérience spéciale de praticiens pour le mode de fixation de la toiture [...] Inutile de dire que cette couverture dont on voit encore beaucoup d'anciennes applications avec des lucarnes en pierre, en Auvergne, dans le Limousin, et dans cette contrée, mais qui est bien peu appliquée à l'état neuf, donne un caractère très particulier à l'édifice. $~^{75}$

\section{8 : Château du Doux}

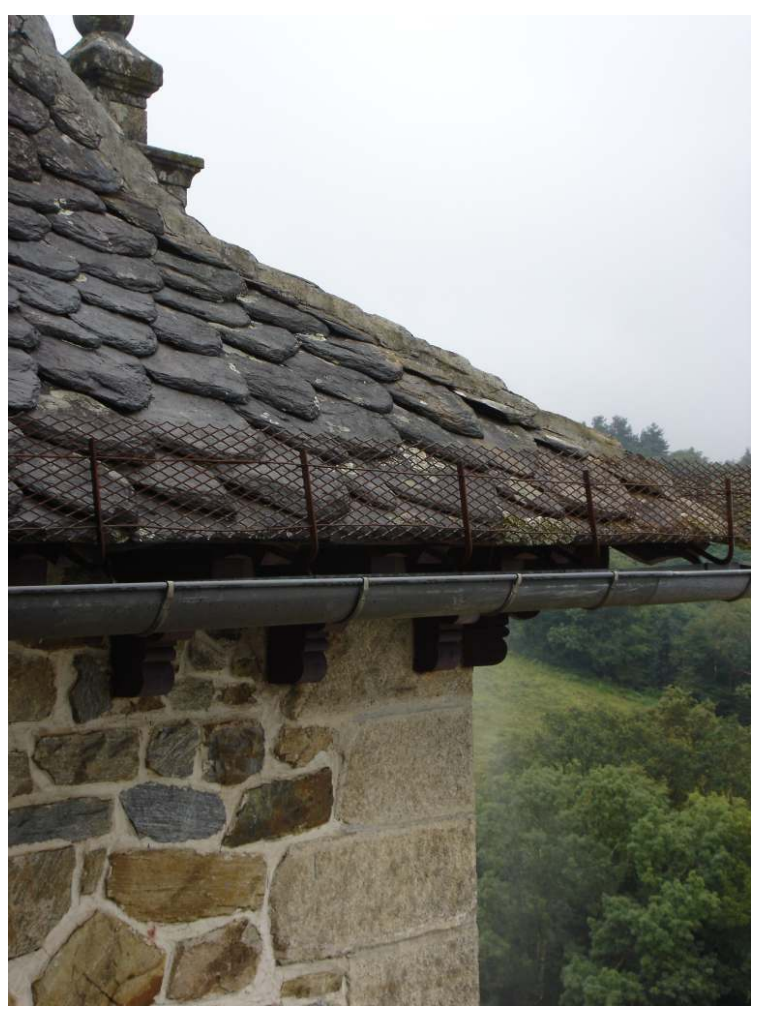

1908. Jean-Louis Pascal. Détails. Des matériaux locaux et des techniques régionales : l'usage de dalles schisteuses pour la toiture

Cl. auteur 
III. 9 : Toiture d'une petite terrasse en plein air à l'angle ouest de la façade sud du château du Doux

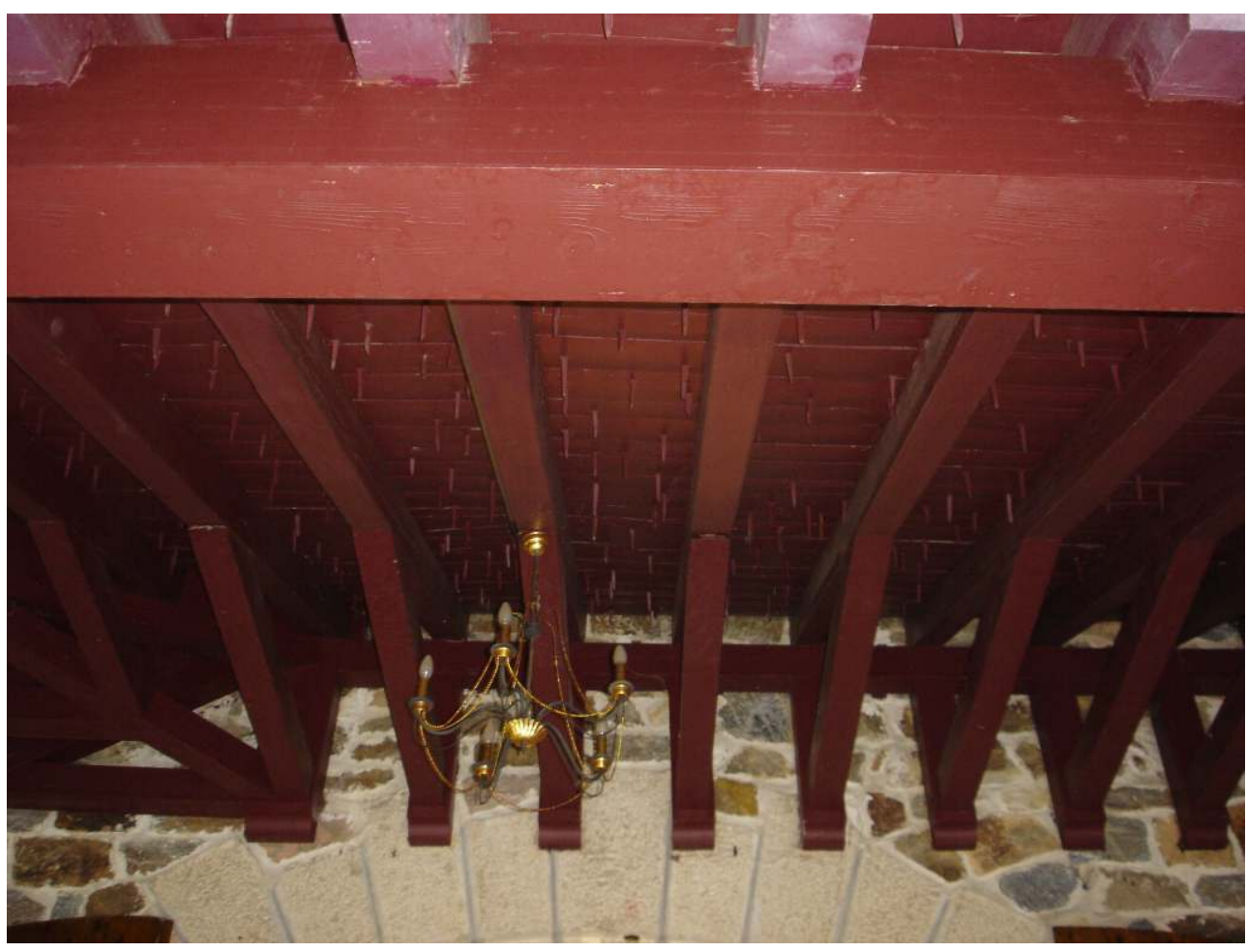

Jean-Louis Pascal. 1908. Détail du voligeage

$\mathrm{Cl}$. auteur

43 On retrouve ici le souci d'employer les matériaux du pays, les savoir-faire de la région, la volonté d'insérer l'architecture dans le paysage, tout ce que le régionalisme a repris du discours rationaliste. La volonté de traduire par la volumétrie la conception du plan relève aussi d'un discours rationaliste. Pascal aurait-il donc été l'un des premiers architectes régionalistes (nous sommes au tout début du siècle) ${ }^{76}$; il a tout du moins exploré cette voie semble-t-il avec bonheur, pour cette « œuvre de prédilection dans sa carrière ", selon ses propres termes.

Les matériaux pour la plus grande partie ont été extraits dans la propriété, à l'exception de la pierre de taille dite " de la Bitarelle ", extraite à cinq kilomètres de là, de constitution granitique, ayant servi pour les angles, les encadrements de fenêtres, les lucarnes et en général pour la pierre d'appareil. C'est le sable de carrière qui a servi pour la confection des mortiers, avec des chaux du Teil et des ciments de la contrée. On retrouve notamment la forme des lucarnes du château du Doux à quelques kilomètres de là, à Beaulieu, dans une maison du XVIII ${ }^{\mathrm{e}}$ siècle, la maison du Bessol ${ }^{77}$, transformée en office du tourisme, dont les lucarnes proviendraient de l'ancienne abbaye de Saint-Pierre, à Beaulieu.

« Si les architectes classiques continuent à ordonner les pièces symétriquement, à accentuer les axes, les admirateurs du Moyen Âge greffent sur le bâtiment principal des annexes qui contiennent l'escalier, les services, projettent hors du bloc un petit salon, un fumoir, le vestibule, et couvrent chaque partie de toits variés ${ }^{78}$, dit Louis Hautecoeur. C'est ce que fait Pascal, lorsqu'il conçoit le plan de son château, groupant par exemple dans la partie de l'entrée au nord autour du vestibule, un parloir et une salle de billard. Un grand hall, orné d'une cheminée de quatre mètres de large, sert de pivot central, sur lequel ouvrent toutes les pièces principales du 
rez-de-chaussée et, par le grand escalier de bois à corbeaux de pierre, sur celles du premier étage. Le souvenir des grandes salles médiévales et l'exemple des halls anglais avaient incité certains propriétaires à imiter cette disposition. Le comte d'Orsay (1801-1852), qui avait longtemps vécu à Londres serait, d'après Arsène Houssaye dans ses Confessions, le premier qui, en 1849, aurait introduit à Paris ces salles qui « servent à la fois de salon, d'atelier, de cabinet de travail, de fumoir, de serre, avec des divans, des lits de repos, des hamacs, tout dans tout ». À l'ouest, au deuxième étage, la grande baie du château du Doux, celle qui offre la meilleure vue sur plusieurs chaînes de montagnes, éclaire la bibliothèque du propriétaire (ill. 6). C'est une baie rectangulaire, tripartite, divisée par des meneaux de pierre granitique et surmontée d'un fronton triangulaire. Elle donne à cette façade l'austérité de certaines architectures anglo-saxonnes. La grande baie en plein cintre à meneaux et à petits carreaux, qui donne le jour au grand escalier sur la façade est (ill. 7), est elle aussi inspirée de l'architecture anglaise. Pascal d'en installer partout pour pouvoir y faire de grands feux de bois. Chaque pièce a une cheminée différente. Les grands vides des cheminées béantes ont donné lieu à d'amusantes recherches de briquetages encadrant des plaques de fonte anciennes dans la salle à manger, le salon, le billard transformé en bar, la bibliothèque. Pour la chambre des parents dont les deux fenêtres donnent au premier étage, au sud, sur la vallée de la Dordogne, Pascal a fait réaliser une magnifique marqueterie de bois pour la cheminée centrale enserrée entre deux belles armoires. bascules et de consoles, pour des combles énormes, notamment sur la partie principale du château, avec un terrasson au sommet, ne fut pas aisée ; la recherche de bois fut aussi difficile dans cette contrée de châtaigniers, bois creux. L'architecte dut aller jusqu'à Bordeaux $^{79}$ où les ressources du port lui fournirent des sapins de Pologne - ceux d'Auvergne sont secs et cassants - et des chênes de Hongrie (en France, dès que les chênes avaient atteint une dimension suffisante pour les traverses de chemin de fer, on les abattait) pour réaliser des poutres de huit mètres et plus, notamment pour la cour de pansage. De beaux noyers de France, mêlés à des noyers d'Amérique donnèrent les lambris de menuiserie de grande hauteur de la salle à manger. Des pitchpins, du redwood de Californie, se prêtèrent à des recherches de mobiliers de service, et diverses essences de bois de choix complétèrent des combinaisons de plafonds à poutres et solives apparentes ${ }^{80}$.

Pascal a donc puisé à différentes sources pour construire ce château : l'architecture de la Renaissance française avec ses fenêtres à meneaux, ses lucarnes coupant la ligne du toit, ses hautes cheminées, l'architecture anglaise avec l'adoption d'un plan dissymétrique, engendrant une volumétrie pittoresque avec des toits séparés, la forme de certaines baies, la présence du grand hall traversant, les pans de bois de l'architecture normande poncif de l'architecture balnéaire et des maisons «à la campagne». Il a adopté une logique «pré-fonctionnelle » en traduisant par les volumes extérieurs la composition du plan et c'est sans doute d'abord l'architecte rationaliste, dans sa volonté d'adaptation au 
site : matériaux, climat, usages locaux - et par là même régionaliste -, employant d'autre part toutes les dernières innovations en matière d'hygiène et de confort, qu'il faut voir ici.

Dans la revue Art et Décoration, « revue où rationalisme et pittoresque s'additionnèrent au rustique, voire au régionalisme ${ }^{81}$ ", René Blum écrivit un article consacré à la villa Natacha $^{82}$ d'Henri Sauvage et Charles Sarazin (1873-1950) à Biarritz; « il y recommandait à l'architecte chargé de dessiner une maison de campagne de n'obéir ni à des "règles établies" ni de "suivre sa seule inspiration"; il devait avant tout se préoccuper de l'usage et de l'implantation sur le terrain. "Il lui fallait en outre respecter autant que possible la physionomie du pays, son esthétique propre et le caractère général de ses habitations. De plus, il devait répondre à certaines nécessités économiques en utilisant les matériaux en usage dans la région" " ${ }^{83}$. C'est aussi sous le rapport de l'intégration au site que Charles Saunier, dans la même revue, louait les deux maisons, les "Sablons » et les "Oyats », que Louis Bonnier (1856-1946) avaient bâties en 1894 à Ambleteuse, station balnéaire du Boulonnais: «Il s'est plu à perpétuer le caractère de l'architecture locale tout en l'adaptant à des besoins nouveaux ${ }^{84}$. »

C'est ce que Pascal s'était appliqué à faire à Altillac; il avait perpétué le caractère de l'architecture locale en ayant recours aux formes de l'habitat régional pour le toit et les lucarnes, il avait utilisé la pierre de taille de la région, la Bitarelle, le pitchpin et le redwood de Californie débarqués à Bordeaux, la dalle schisteuse d'Auvergne. "Le programme qu'on m'avait donné était de faire une maison qui fût bien du pays [avait écrit Pascal] ; je fis de mon mieux pour qu'elle fût bien aussi dans le paysage $»^{85}$. Pascal était aussi un architecte régionaliste.

\section{NOTES}

1. «En dehors de ma fonction officielle qui me semble pouvoir m'être retirée sans possibilité de résistance, je ne peux plus gagner assez pour payer le loyer que j'ai. Le bon sens depuis longtemps, depuis quinze ou dix-huit ans, aurait dû me forcer à déménager et la conviction tenace que je devais partir, celle qu'aujourd'hui encore je subis, et naturellement plus justement, plus logiquement que précédemment, partir pour l'audelà, bientôt, demain, m'a fourré dans cette impasse d'une fin, brusquement, de misère qui m'a terrifié. » Lettre de Jean-Louis Pascal, alors qu'on lui a "retiré » son poste à la Bibliothèque nationale, probablement à sa nièce, Jeanne-Julie Pascal mais non envoyée. Bibl. nat. de France, dép. des Manuscrits, N.a.fr. 25093, p. 232.

2. La salle de concerts du Casino de Monte Carlo construite entre mai 1878 et janvier 1879 par Charles Garnier fut modifiée en 1897 contre son gré par le nouvel architecte de Monte Carlo, Henri Schmidt. Jean-Michel Leniaud, Charles Garnier, Paris, 2003, éd. du Patrimoine, 175 p., p. 73-79.

3. B.E.B.A., Ms 745, Lettres de l'architecte Jean-Louis Pascal à Louise Garnier, lettre $n^{\circ} 38,24$ mars 1912. 
4. Georges Seure, préface aux Monuments Antiques, p. 2-3, cité par Marie-Françoise Billot, dans » Recherches aux $\mathrm{XVIII}^{\mathrm{e}}$ et $\mathrm{XIX}^{\mathrm{e}}$ siècles sur la polychromie de l'architecture grecque ", Paris - Rome - Athènes, Le voyage en Grèce des architectes français aux XIX et XX ${ }^{e}$ siècles, E.N.S.B.A., 1982, 436 p., p. 61-125.

5. Charles Garnier et Auguste Ammann, L'Habitation humaine, Paris, Librairie Hachette, 1892, 895 p., p. 812, cité par Jean-Michel Leniaud, Charles Garnier, op. cit., p. 107.

6. L'atelier Gilbert fut créé par Pierre Jules Delespine (1756-1825) en 1800 ; Guillaume Abel Blouet (1795-1853) en prend la tête de 1825 à 1853, remplacé par Jacques Félix Duban (1797-1870) pendant l'expédition de Morée (février 1829-mars 1830). Jacques Émile Gilbert remplace Abel Blouet en 1853. Quand il renonce à l'enseignement en 1855, une pétition émanant des élèves de l'atelier - dont Jean-Louis Pascal - demande à Félix Duban de le remplacer. Celui-ci décline la proposition, acceptée par Charles Auguste Questel (1807-1888) qui fait de cet atelier le plus grand atelier de la fin du Second Empire. C'est le même atelier que Pascal dirigera pendant près de cinquante ans, de 1872 à sa mort, devenant ensuite l'atelier Recoura-Duquesne.

7. Charles-Marie Widor, Notice sur la vie et les travaux de M. Jean-Louis Pascal, 4 juin 1837 - 16 mai 1920. Lue dans la séance publique annuelle du 4 décembre 1920, Paris, Firmin-Didot, 1920, 16 p., p. 7.

8. Je remercie vivement Michel Gallet pour les nombreux entretiens qu'il m'a accordés au cours desquels il m'a raconté la vie à l'atelier Pascal.

9. Ses interventions pour aider ses amis, ses élèves, sont extrêmement nombreuses.

10. B.n.F., dp. Mss, N.a.fr. 25093, lettre d'Édouard Arnaud à Jean-Louis Pascal, 14 avril 1912, p. 13.

11. L'atelier Redon (dont faisaient partie Albert Laprade (1883-1978), Louis Hippolyte Boileau, Michel Roux-Spitz (1888-1957), transfuge de l'atelier Pascal) était aussi situé rue Mazarine; il y avait une certaine concurrence entre les deux ateliers; un jour «les Pascal » firent le siège de l'atelier Redon dont la défense fut assurée par Boileau. Propos rapportés par Michel Gallet.

12. Marc Saboya, Robert Coustet, Bordeaux, le temps de l'histoire. Architecture et urbanisme au XIXe siècle (1800-1914), Bordeaux, éd. Mollat, 1999, 271 p., p. 125.

13. Jean-François Champollion aménage au $n^{\circ} 28$ de la rue Mazarine en juillet 1821 dans un logement situé au-dessus de celui de son frère, Jacques-Joseph, à quelques dizaines de mètres de l'Institut. Jean Lacouture, Champollion, une vie de lumières, Paris, Éd. Grasset et Fasquelle, 1988, 529 p., p. 282.

14. Jean Formigé, Notice sur la vie et les travaux de M. J.-L. Pascal, 4 juin 1837-16 mai 1920, Institut de France, Académie des beaux-arts, 12 novembre 1921, 12 p., p. 5.

15. Amateur éclairé qui possédait une galerie à Paris, 47 rue Le Peletier $\left(9^{\mathrm{e}}\right)$, et qui avait exposé les peintres nabis en 1891 et Vincent Van Gogh dès 1894. « Comme Samuel Bing, il présentait et commercialisait les objets exotiques et les réalisations d'artistes décorateurs novateurs. » Jean-Baptiste Minnaert, Henri Sauvage, Paris, éd. Norma, I.F.A./Cité de l'Architecture et du Patrimoine, 2002, 408 p., p. 28.

16. Maurice Culot, Lise Grenier (dir.), Henri Sauvage, 1873-1932, catalogue d'exposition, Bruxelles, Paris, Archives d'architecture moderne / S.A.D.G., 1976.

17. Le samedi 6 novembre 1897.

18. M. Michel Gallet qui en possède un exemplaire a eu la gentillesse de me le montrer. 
19. B.n.F., dp. Mss, N.a.fr. 25093, p. 194-198, «Jubilé de M. Pascal », samedi 6 novembre 1897, compte-rendu de Paul Wallon, extrait du journal d'architecture, 6 p., p. 3.

20. Ce livre, exemplaire unique, peut être consulté à la réserve du département des estampes de la Bibliothèque nationale sous la cote : $\mathrm{Pd} 122$, réserve, in- $8^{\circ}$.

21. En 1897, c'est Charles Victor Bartaumieux (1832-1907) qui est président de l'association.

22. Jean-Louis Pascal, « De l'organisation de l'École nationale et spéciale des beaux-arts », R.G.A.T.P., $\mathrm{n}^{\circ} \mathrm{XXXI}$, 1874, p. 221-230.

23. B.E.B.A., Ms 744, Lettres de l'architecte Jean-Louis Pascal à Charles Garnier, op. cit., lettre $n$ - 139, 22 avril 1882. Pascal propose le nom de Gustave Moreau à Garnier pour le siège de Karl Ernest Lehmann, à l'Institut, en 1882, quand Garnier pense, lui, à Henner, Boulanger ou Lefebvre.

24. Arch. nat., $\mathrm{AJ}^{52} 495$, procès-verbaux du conseil supérieur de l'enseignement de l'École des beaux-arts, séance du 24 mai 1898.

25. Son directorat dure de 1866 à 1867.

26. Henri Lapauze, Histoire de l'Académie de France à Rome, Paris, 1924, tome II : 1802-1910, 595 p., p. 376.

27. Lettre de Paul Baudry à ses parents, Rome, 9 mars 1851, cité par Charles Ephrussi, Paul Baudry, sa vie et son œuvre, Paris, éd. Ludovic Baschet, 1887, 328 p., p. 74.

28. Son premier directorat dure de 1867-1872.

29. Henri Lapauze, Histoire de l'Académie de France à Rome, op. cit., p. 381-382.

30. Il s'agit du peintre Diogène Ulysse Napoléon Maillart ou Maillard (1840-1926), élève de Laemlein, Cornu et L. Cogniet, entré à l'E.B.A. en 1860, $1^{\mathrm{er}}$ grand prix de Rome en 1864 avec Homère dans l'île de Cyros. Émile Bellier de la Chavignerie, Louis Auvray, Dictionnaire général des artistes de l'École française depuis l'origine des arts du dessin jusqu'à nos jours, architectes, peintres, sculpteurs, graveurs et lithographes, Paris, H. Loones, 1885, vol. 2, p. 11.

31. Henri Lapauze, Histoire de l'Académie de France à Rome, op. cit., p. 383-384.

32. Arch. nat., $\mathrm{AJ}^{52} 495$, rapport à monsieur le ministre de l'instruction publique, des cultes et des beaux-arts sur les travaux envoyés à Rome par les pensionnaires en 1871.

33. La Villa Madame, villa suburbaine située au pied du Monte Mario dans la campagne romaine, fut commencée à la fin du pontificat de Léon X pour le cardinal Jules de Médicis élu pape sous le nom de Clément VII (1523-1534), par Raphaël - Jules Romain selon Vasari ; la galerie des Offices de Florence en avait deux plans attribués à Antonio da Sangallo le Jeune. Bénard s'y appuie pour attribuer la construction à l'architecte florentin choisi par le cardinal Jules de Médicis. Vasari se serait trompé.

34. Arch. nat., $\mathrm{AJ}^{52} 495$, rapport à monsieur le ministre de l'instruction publique, des cultes et des beaux-arts sur les travaux envoyés à Rome par les pensionnaires en 1871.

35. Jean-Louis Pascal, « De l'organisation de l'École nationale et spéciale des beaux-arts », R.G.A.T.P., ${ }^{\circ} \mathrm{XXXI}, 1874$, p. 229.

36. Pascal est nommé à l'Institut le 3 mai 1890 au fauteuil de Jules André. Archives de l'Institut, registre des procès-verbaux, $2 \mathrm{E} 18$.

37. Archives de l'Institut, 2E18. Procès-verbaux de l'Académie. Rapport de Pascal au nom de la section d'architecture, séance du 14 novembre 1890, en réponse à la lettre du 
ministre des beaux-arts au secrétaire perpétuel, à propos du règlement de l'École de Rome.

38. Archives de l'Institut, 5E63. Correspondance de l'année 1891. Lettre du directeur des beaux-arts G. Larroumet au président de l'Académie des beaux-arts.

39. Charles Ephrussi, Paul Baudry, sa vie et son œuvre, op. cit., p. 167.

40. « Nouvelles. Paris. Jury des récompenses à l'Exposition », La Construction moderne, $\mathrm{n}^{\circ} 4$, 1888-1889, 8 juin 1889, p. 420. "Nouvelles. Paris. Jury des récompenses à l'Exposition universelle ", La Construction moderne, $\mathrm{n}^{\circ}$ 4, 1888-1889, 29 juin 1889, p. 456.

41. Le président du bureau était Émile Vaudremer, le vice-président: le Dr. Petrus Josephus Hubertus Cuypers, architecte des musées de l'État à Ruremonde, Pays-Bas, et le secrétaire : H. Mayeux.

42. Jean-Louis Pascal, Rapport sur la classe 10, architecture, à l'exposition universelle internationale de 1900, Paris, 1902, $21 \mathrm{p}$.

43. Vue par Charles Garnier en 1892 dans L'Habitation humaine: comme « une des formes, ou du moins, des exigences du progrès » cité dans Jean-Michel Leniaud, Les Bâtisseurs d'avenir. Portraits d'architectes. XIX'-XX' siècle, Paris, 1998, Fayard, 503 p., p. 112.

44. La Semaine des constructeurs, novembre $1887,2^{\mathrm{e}}$ série, $2^{\mathrm{e}}$ année, cité dans Jean-Pierre Épron, Comprendre l'Éclectisme, Paris, éd. Norma, 1997, 357 p., p. 171 et note 107 p. 346.

45. Jean-Louis Pascal, « Exposition universelle de Vienne », R.G.A.T.P., $\mathrm{n}^{\circ} \mathrm{XXX}$, 1873, p. 204.

46. Pascal participera d'ailleurs à la création des premières écoles régionales d'architecture. Il écrit à Cassien-Bernard, le 26 avril 1903, « en rentrant de Marseille où nous étions pour tenter un essai d'École régionale d'architecture ». Arch. adm. B.n.F., 2011/001/10010.

47. Cette société était présidée par Georges Picot (1838-1909), le fondateur de la société des Habitations à bon marché et par l'architecte Anatole de Baudot (1834-1915). JeanClaude Vigato, L'Architecture régionaliste, France, 1890-1950, Paris, Norma éd., 1994, 390 p., p. 22.

48. Jean Lahor, Les Habitations à bon marché et un Art nouveau pour le peuple, Paris, Librairie Larousse, s.d. [1904], cité par Jean-Claude Vigato, L'Architecture régionaliste, France, 1890-1950, op. cit., p. 24.

49. Jean-Louis Pascal, « Exposition universelle de Vienne », R.G.A.T.P., $\mathrm{n}^{\circ} \mathrm{XXXI}, 1874$, p. 63.

50. Ibid., p. 273.

51. Jean-Louis Pascal, Rapport sur la classe 10, architecture à l'Exposition Universelle internationale de 1900, op. cit., p. 21.

52. Charles Garnier, dans L'Habitation humaine qu'il publie en 1882 avec Auguste Ammann, attribuait ce phénomène d'uniformisation croissante de l'habitat à la production industrielle des matériaux, au perfectionnement croissant des règles d'urbanisme tendant à l'uniformisation, à la densification des populations qui oblige à construire en hauteur, et au développement des principes hygiénistes. Jean-Michel Leniaud, Charles Garnier, op. cit., p. 107.

Les principes d'Henri Labrouste qui exigeait de ses élèves des caractères distincts pour les constructions à élever dans le Nord ou le Midi et qui enseignait à composer un projet d'architecture en se préoccupant de savoir si la province où il devait être bâti produisait les matériaux nécessaires à l'édification, semblent bien loin. 
53. Le Grand palais.

54. Jean-Louis Pascal, Rapport sur la classe 10, architecture, à l'Exposition Universelle internationale de 1900, op. cit., p. 11-13.

55. Ibid., p. 21.

56. Les architectes provinciaux avaient demandé en 1889 un diplôme d'architecte reconnu par l'État qui ne fût pas un simple diplôme scolaire; une commission administrative avait été créée pour la circonstance : «Larroumet, directeur des BeauxArts ouvre la première séance le 27 janvier 1890 . Sont présents : Charles Garnier, Anatole de Baudot, Chevallier, le créateur de l'Association provinciale, Pascal qui remplace Jules André mort quelques jours avant la réunion, Bourdais (1835-1915), l'auteur du Trocadéro avec Davioud, Achille Hermant, César Daly, Charles Lucas, l'historiographe des architectes, et Journoud, l'architecte de Lyon ». Jean-Pierre Épron, Comprendre l'éclectisme, op. cit., p. 50. Lorsque l'on posa la question:»Y-a-t-il lieu d'instituer le diplôme obligatoire? », la commission, dont Pascal, vota «non» par quinze voix contre trois : celles des architectes provinciaux: Lucien Lefort (1850-1916), Journoud (1828-1897) et Chevallier. Jean-Claude Vigato, L'Architecture régionaliste, France, 1890-1950, op. cit., p. 16.

57. Jean-Louis Pascal, «Les Écoles Régionales d'Architecture", L'Architecture, 1904, p. 393-395.

58. Ibid., p. 394.

59. Ibid., p. 395.

60. Arch. nat., $\mathrm{AJ}^{52} 21$, conseil supérieur d'enseignement de l'École des beaux-arts, procèsverbaux, séance du 10 avril 1908.

61. En 1903, les décrets et arrêtés créant huit écoles régionales avaient été promulgués.

62. Arch. nat., $\mathrm{AJ}^{52} 21$, conseil supérieur d'enseignement de l'École des beaux-arts, procèsverbaux, séance du 27 juin 1908.

63. La commission était constituée de : Léon Bonnat (président); Vaudremer, Pascal, Nénot, Bernier, Blavette, Roujon, Richer, Lemonnier, Bourlet, Pillet, Masson, Monduit, Magne, Boeswillwald architectes. Maneuvrier et Cassagnade professeurs d'histoire et de sciences, Collin et Allar professeurs au cours des trois arts, Daumet président du jury d'architecture, Lelong délégué des Écoles régionales, Bonnier et Baschet secrétaires adjoints du conseil. Ibid., séance du 24 juillet 1909.

64. Ibid., 18 février 1910.

65. Ibid., séance du 3 mai 1913.

66. «Concours publics. Concours international pour le plan de l'université de Californie, à Berkeley ", L'Architecture, 1898, $11^{\mathrm{e}}$ année, $\mathrm{n}^{\circ}$ 4, samedi 22 janvier 1898, p. 30-31.

67. Le château du Doux entouré d'un parc de trois hectares, est situé au sud du département de la Corrèze, à la limite du Lot, à $45 \mathrm{~km}$. de Brive et de Tulle. C'est actuellement un hôtel-restaurant.

68. Pascal avait demandé des honoraires s'élevant à $10 \%$, et non à $5 \%$ comme c'était la règle, du coût total des travaux. Il estimait que sa notoriété le lui permettait. Propos tenu à André Gallet et rapporté par son fils Michel Gallet, que je remercie.

69. Jean-Louis Pascal, « Le Domaine du Doux. Commune d'Altillac (Corrèze) », L'Architecte, février 1908, p. 9-15. 
70. B.E.B.A., Ms 745, Lettres de l'architecte Jean-Louis Pascal à Louise Garnier, op. cit., lettre n - 27, Le Doux, commune d'Altillac, jeudi 6 août 1908.

71. Jean-Louis Pascal, « Le Domaine du Doux. Commune d'Altillac (Corrèze) », L'Architecte, op. cit., p. 9-15.

72. Cet espace a été remplacé par une piscine.

73. Jean-Louis Pascal, « Le Domaine du Doux. Commune d'Altillac (Corrèze) », L'Architecte, op. cit., p. 10-11.

74. B.E.B.A., Ms 745, Lettres de l'architecte Jean-Louis Pascal à Louise Garnier, op. cit., lettre n -32, 4 mars 1910.

75. Jean-Louis Pascal, « Le Domaine du Doux. Commune d'Altillac (Corrèze) », L'Architecte, op. cit., p. 12.

76. Pour l'historien François Loyer, la première œuvre régionaliste serait l'hôtel de ville de Loos-lez-Lille construit en 1884 par l'architecte lillois Louis-Marie Cordonnier (1854-1940). François Loyer, "Ornement et caractère ", Le siècle de l'éclectisme. Lille 1830-1930, Bruxelles, Archives d'Architecture moderne, 1979, p. 65-104.

77. C'est le lieu de naissance du général Dufaure du Bessol (1828-1908) auxiliaire dévoué du général Faidherbe à Sedan en 1870.

78. Louis Hautecoeur, Histoire de l'Architecture classique en France, la fin de l'architecture classique (1848-1900), vol. VII, Paris, Picard, 1957, 575 p., p. 248.

79. Pascal avait travaillé dans les années 1870 à la construction de la faculté de médecine de Bordeaux et la recherche de bois pour le mobilier l'avait orienté vers un industriel de Bordeaux : Carde, qui en 1900 était venu à Paris, faire, pour la Société des artistes, la charpente de ce que l'on appela le Salon de Breteuil.

80. Les entrepreneurs qui ont travaillé au Doux avec Pascal sont: Gasquet, entrepreneur d'Aurillac qui a réalisé la couverture de pierre, Bussière, de Bretenoux-Biars, entrepreneur de maçonnerie, Bombézy (paratonnerres), le quincailler Bricard, de Paris, la maison Geneste-Herscher (radiateurs), Nicora pour la fumisterie, la maison Allioli frères, de Paris, (peinture), un entrepreneur parisien, Lamardelle, pour les enduits. La plomberie, l'installation des cuisines et des salles de bain fut confiée à une maison bordelaise qui avait une succursale à Paris, la maison Genvré.

81. Jean-Claude Vigato, L'Architecture régionaliste, France, 1890-1950, op. cit., p. 41.

82. Sur la villa Natacha construite en 1905-1907 par l'agence immobilière qu'Henri Sauvage et Charles Sarazin avaient créée à Biarritz, voir Jean-Baptiste Minnaert, Henri Sauvage, op. cit., p. 135-143.

83. Jean-Claude Vigato, L'Architecture régionaliste, France, 1890-1950, op. cit., p. 40, citant René Blum, «Une maison de campagne par MM. Sauvage et Sarazin », Art et Décoration, $2^{\mathrm{e}}$ semestre 1908, t. XXIV, p. 163-172.

84. Charles Saunier, "Deux cottages de Louis Bonnier ", Art et Décoration, $1^{\mathrm{er}}$ semestre 1907, t. XXI, p. 187-192, cité par Jean-Claude Vigato, L'Architecture régionaliste, France, 1890-1950, op. cit., p. 40.

85. Jean-Louis Pascal, « Le Domaine du Doux. Commune d'Altillac (Corrèze) », L'Architecte, op. cit., p. 12. 


\section{RÉSUMÉS}

Jean-Louis Pascal connaît une fin de vie triste et il sombre dans la mélancolie. Son départ de la Bibliothèque, en 1912, est douloureux, même s'il laisse la place à Ernest Recoura, un de ses élèves préférés, qu'il choisit pour lui succéder. Son rôle de chef d'atelier, qu'il exerce depuis 1872 , le passionne cependant toujours autant ; son charisme et la réussite de ses élèves au grand prix de Rome attire les candidats et beaucoup d'élèves américains, suisses, autrichiens viennent suivre ses cours à l'atelier. Il participe au début du siècle à la création des écoles régionales d'architecture et s'impose dans le débat architectural, comme il l'avait fait tout au long de sa vie à travers les nombreux articles parus dans la presse spécialisée qui révèlent un réel talent de plume. Pascal trouvait l'uniformité des immeubles construits à Paris, due aux règles imposées par le préfet Haussmann, "écœurante" ; il s'insurge aussi, comme Charles Garnier, contre l'internationalisation des styles et prône une architecture utilisant les matériaux locaux, s'adaptant au climat et aux usages régionaux. Il en donnera l'exemple en construisant au tout début du XX $\mathrm{XX}^{\mathrm{e}}$ siècle, pour un couple franco-américain, une belle demeure corrézienne, le château du Doux, qu'il considère comme une de ses plus belles réalisations.

Getting depressed, Jean-Louis Pascal spends a sad end of life. His leaving of the National Library, in 1912, is difficult, even he is succeeded by Ernest Recoura, one of his favorite students he chose to fill his place. He is still passionate about his role as a workshop leader he used to be since 1872 ; his charisma and his students success for winning the Grand Prix de Rome attract many foreign students, coming from America, Switzerland and Austria, and eager to attend his tuitions. At the beginning of the new century, he takes part in the creation of the regional school of architecture and becomes important in the architectural debate, as he used to do throughout his all life writing many papers published in the specialist press, which shows real writing skills. Pascal considered the buildings uniformity, due to the works carried out by the Préfet Haussmann, as "disgusting". As Charles Garnier did, he stands against styles internationalization and claims for an architecture using local materials, respecting the climate and the regional habits. He will give a proof of this requirement at the early $\mathrm{XX}^{\text {th }}$ century, for a mansion he designed for a FrancoAmerican couple : the castle of the Doux he considered as one of his most brilliant realisations.

Jean-Louis Pascal verbringt seine letzten Lebensjahre in düsterer und melancholischer Stimmung. Sein Abschied von der Bibliothèque Nationale im Jahre 1912 gestaltet sich als schwierig, obwohl er einem seiner Lieblingsschüler, Ernest Recoura, sein Amt übergibt, welchen er selbst für seine Nachfolge auswählt. Seine Position als Atelierleiter, die er ab 1872 ausübt, verfolgt er jedoch weiterhin mit großer Leidenschaft. Sein Charisma und die Erfolge seiner Schüler beim Grand Prix de Rome ziehen zahlreiche Studenten aus den USA, der Schweiz und Österreich an, die in seinem Atelier Kurse belegen. Auch ist Pascal zu Beginn des 20. Jahrhunderts an der Gründung regionaler Architekturschulen beteiligt und weiterhin rege an architekturtheoretischen Debatten beteiligt, wie er sich auch zeitlebens in zahlreichen in der Fachpresse erschienenen Artikeln äußert, die sein schriftstellerisches Talent verdeutlichen. Pascal beurteilt die Gleichförmigkeit der in Paris gemäß den Vorgaben des Präfekten Haussmann errichteten Gebäude als "abstoßend", lehnt sich ebenso wie Charles Garnier gegen eine Internationalisierung der Stile auf und tritt vielmehr dafür ein, lokale Materialien $\mathrm{zu}$ verwenden, die sich dem 
jeweiligen regionalen Klima und den Nutzungsgewohnheiten der Einwohner anpassen. Pascal selbst liefert dafür mit dem am Anfang des 20. Jahrhunderts für ein franco-amerikanisches Ehepaar errichteten Château de Doux im Departement Corrèze eines der eindrücklichsten Beispiele.

\section{INDEX}

Index chronologique : XXe siècle, époque contemporaine

Mots-clés : régionalisme

Schlüsselwörter : Regionalismus

Keywords : regionalism

\section{AUTEUR}

\section{ANNE RICHARD-BAZIRE}

Anne Richard-Bazire est docteur de l'École pratique des hautes études (section des sciences historiques et philosophiques), diplômée en histoire de l'art contemporain et en archéologie égyptienne. Elle est actuellement chargée du cours de synthèse de l'Histoire de l'architecture occidentale, $\mathrm{XIX}^{\mathrm{e}}-\mathrm{XX}^{\mathrm{e}}$ siècles, à l'École du Louvre. Elle a rédigé différents articles : «L'habitat néo-gothique à Paris au XIX ${ }^{\mathrm{e}}$ siècle ", La demeure médiévale à Paris, Étienne Hamon et Valentine Weiss (dir.), Paris, 2012, Archives Nationales, p. 250-257 ; « La salle Ovale ou l'histoire des dysfonctionnements dans les chantiers parisiens du XIX ${ }^{\mathrm{e}}$ siècle à travers l'exemple de la Bibliothèque nationale ", La Collégialité et les dysfonctionnements dans la décision administrative, JeanMichel Leniaud et François Monnier (dir.), Paris, 2011, École Pratique des Hautes Études, p. 103-112; " Jean-Louis Pascal ou le respect du patrimoine », article mis en ligne sur le site "Archicab", le 4 décembre 2011, http://www.jeanfrancoiscabestan.com/bnfrichelieu.html ; « Le concours pour la reconstruction de l'Hôtel de Ville de Paris (1872-1873), un échec pour l'architecte Jean-Louis Pascal », Livraisons d'histoire de l'architecture, n 19, 2010, p.111-134 ; « L'escalier d'honneur de la Bibliothèque nationale de la rue de Richelieu ", Sites et Monuments, $\mathrm{n}^{\circ}$ 207, oct.-nov.-déc. 2009, p. 22-24 ; «La salle Ovale », Revue de la Bibliothèque nationale de France, $\mathrm{n}^{\circ}$ 30, 2008, 96 p., p. 32-39 ; « La faculté de médecine et de pharmacie de Bordeaux par Jean-Louis Pascal (1876-1888 et 1902-1922) », Livraisons d'histoire de l'architecture, $\mathrm{n}^{\circ} 13,2007, \mathrm{p} .105-120$; « Le ministère de l'agriculture de la rue de Varenne par Emmanuel Brune, l'alliance de la science et de l'art », Livraisons d'histoire de l'architecture, $\mathrm{n}^{\circ}$ 8, 2004, p. 81-94; « Le parvis de Notre-Dame » et « Le square Jean XXIII », Autour de Notre-Dame, sous la dir. de Alain Erlande-Brandeburg, Jean-Michel Leniaud, François, Christian Michel, Paris, D.A.A.V.P., 2003, 269 p., p. 240-242 et 243-245 ; « Les réalisations de Pascal », et « L'École des beaux-arts » avec Fabienne Doulat, Les bibliothèques parisiennes, architecture et décor, sous la dir. de Myriam Bacha et Christian Hottin, Paris, D.A.A.V.P., 2002, 272 p., p. 115-117 et 132-134; « Un siècle de réflexion sur la construction des bibliothèques ", Des palais pour les livres, Labrouste, Sainte-Geneviève et les bibliothèques, sous la dir. de Jean-Michel Leniaud, Paris, éd. Maisonneuve \& Larose, 2002, 189 p., p. 58-69 ; « Jean-Louis Pascal et la création de la salle des périodiques de la Bibliothèque nationale (1883-1936) », Livraisons d'histoire de l'architecture, $\mathrm{n}^{\circ} 1$, 2001, p. 105-125. Elle a dirigé avec Martin Drouin : La Sélection patrimoniale, Québec, Éditions MultiMondes, coll. Cahiers de l'Institut du patrimoine de l'UQAM, n 13, 2011, 388 p. Elle a participé en octobre 2001 au colloque international organisé par la Bibliothèque Sainte-Geneviève à Paris : « Henri Labrouste et la Bibliothèque Sainte-Geneviève, Histoire et 
actualité », en octobre 2009 au colloque : « Victor Laloux, un architecte dans sa ville » organisé à Tours par le Conseil général d'Indre-et-Loire, en juillet 2010 aux journées d'études organisées par le Centre-Ledoux-Université Paris I, la Commission du Vieux Paris et l'Association pour la Sauvegarde et la Mise en valeur du Paris Historique, sur le thème : «BNF Richelieu : un projet en questions »; en octobre 2010, elle a été la coordinatrice scientifique avec Martin Drouin de la Sixième Rencontre internationale des jeunes chercheurs en patrimoine sur le thème de la " Sélection patrimoniale », organisée conjointement par l'Université du Québec à Montréal et l'École des Chartes à Paris, sous la direction de Luc Noppen, Lucie K. Morisset (UQAM) et JeanMichel Leniaud (École des chartes). Elle a participé en janvier 2011 aux Journées annuelles de l'équipe Histoire du droit public et de l'administration, EPHE, sous la direction de François Monnier, directeur de l'UR, Jean-Michel Leniaud et Jean-Miguel Pire sur le thème : « Les dysfonctionnements dans la décision administrative ", en novembre 2011 à la journée d'études de l'équipe Histara de l'EPHE, organisée à l'INHA par Jean-François Belhoste, directeur d'études à l'EPHE, et Isabelle Parizet, maître de conférences à l'EPHE, sur le thème : « Architectures bancaires : entre le Beau et l'Utile », en décembre 2012 au colloque international organisé par l'INHA, Le musée d'Orsay et la Maison des centraliens : « William Le Baron Jenney (1832-1907). De l'École centrale de Paris aux gratte-ciel de Chicago ». Elle a réalisé de mars 2012 à janvier 2013 pour le compte de l'OPPIC les recherches historiques préalables à la restauration des galeries Mansart, Mazarine et de la « chambre de Mazarin » à la Bibliothèque nationale Richelieu sous la direction de l'architecte en chef des monuments historiques A. de Saint-Jouan. Adresse électronique : anne.richardbazire@hotmail.com. 